\title{
Distinctive regulatory architectures of germline-active and somatic genes in C. elegans
}

\author{
Jacques Serizay, Yan Dong, Jürgen Jänes, Michael Chesney, Chiara Cerrato, \\ and Julie Ahringer \\ The Gurdon Institute and Department of Genetics, University of Cambridge, Cambridge CB2 1QN, United Kingdom
}

\begin{abstract}
RNA profiling has provided increasingly detailed knowledge of gene expression patterns, yet the different regulatory architectures that drive them are not well understood. To address this, we profiled and compared transcriptional and regulatory element activities across five tissues of Caenorhabditis elegans, covering $\sim 90 \%$ of cells. We find that the majority of promoters and enhancers have tissue-specific accessibility, and we discover regulatory grammars associated with ubiquitous, germline, and somatic tissue-specific gene expression patterns. In addition, we find that germline-active and soma-specific promoters have distinct features. Germline-active promoters have well-positioned +1 and -1 nucleosomes associated with a periodic 10-bp WW signal ( $\mathrm{W}=\mathrm{A} / \mathrm{T})$. Somatic tissue-specific promoters lack positioned nucleosomes and this signal, have wide nucleosome-depleted regions, and are more enriched for core promoter elements, which largely differ between tissues. We observe the 10-bp periodic WW signal at ubiquitous promoters in other animals, suggesting it is an ancient conserved signal. Our results show fundamental differences in regulatory architectures of germline and somatic tissue-specific genes, uncover regulatory rules for generating diverse gene expression patterns, and provide a tissue-specific resource for future studies.
\end{abstract}

[Supplemental material is available for this article.]

Cell type-specific transcription regulation underlies production of the myriad of different cells generated during development. Regulatory elements (i.e., promoters and enhancers) are key sequences that direct appropriate spatiotemporal gene expression patterns, and they can have diverse activities, ranging from ubiquitous to highly cell type specific (Smith et al. 2007; Cusanovich et al. 2018; Andersson and Sandelin 2019; Liu et al. 2019). The composition, activity, and arrangement of regulatory elements define the regulatory grammar that controls patterns of gene transcription across development (Levine 2010; Ong and Corces 2011; Spitz and Furlong 2012; Heinz et al. 2015; Rajendra Sonawane et al. 2017), and mutation or perturbation of their spatial organization can lead to pathologies (Lupiáñez et al. 2016).

Previous studies have provided important and increasingly detailed knowledge of features of transcription regulation in eukaryotes. Different regulatory architectures have been observed, ranging from single promoters to complex structures involving multiple regulatory elements, which can operate redundantly, hierarchically, additively, or synergistically (Herr 1993; Davuluri et al. 2008; Guerrero et al. 2010; Whyte et al. 2013; Bahr et al. 2018; Osterwalder et al. 2018). Work on human cells suggests that housekeeping genes are primarily regulated by a single core promoter, whereas tissue-specific genes rely on additional regulatory elements (Ernst et al. 2011). Moreover, differences in sequence features, patterns of transcription initiation, and nucleosome arrangement characterize promoters with different activities (Lenhard et al. 2012; Haberle and Lenhard 2016). Yet, cell typespecific differences are still not well understood. More comprehensive genome-wide in vivo studies of regulatory grammar would directly address how specific gene expression patterns in different

\section{Corresponding author: ja219@cam.ac.uk}

Article published online before print. Article, supplemental material, and publication date are at http://www.genome.org/cgi/doi/10.1101/gr.265934.120. Freely available online through the Genome Research Open Access option. tissues are achieved and whether expression is governed by distinct regulatory architectures. Caenorhabditis elegans is a powerful system to study tissue-specific regulatory grammar, with its small genome, simple anatomy, and wealth of genomic data (The C. elegans Sequencing Consortium 1998; Gerstein et al. 2010; Ho et al. 2014; Jänes et al. 2018; Kudron et al. 2018). To investigate tissue-specific regulatory grammars, we profiled and compared nuclear transcriptomes and chromatin accessibility in sorted $C$. elegans tissues. Analyses of these rich data sets revealed shared and distinct features of ubiquitous and tissue-specific regulatory architectures.

\section{Results}

Tissue-specific profiling of chromatin accessibility and gene expression in $C$. elegans tissues

To investigate the regulatory chromatin of different cell types and how it relates to gene expression, we developed a procedure to isolate nuclei from individual tissues in C. elegans by expressing GFP tags on the outside of the nuclear envelope using tissue-specific promoters and by isolating labelled nuclei using fluorescent-activated nuclear sorting (see Methods) (Fig. 1A; Supplemental Figs. S1, S2). Here we focus on tissue-specific gene expression using transgenes active in the germline or the four major somatic tissues of $C$. elegans (muscle, hypodermis, intestine, and neurons). To isolate fully differentiated somatic tissues and avoid inclusion of nuclei from embryos, we synchronized animals at the L1 stage then fed them until the population predominantly contained late L4s and young adults without embryos. We obtained nuclei of high purity from each tissue $(97.4 \% \pm 1.27$ SD) (Supplemental

C) 2020 Serizay et al. This article, published in Genome Research, is available under a Creative Commons License (Attribution 4.0 International), as described at http://creativecommons.org/licenses/by/4.0/. 

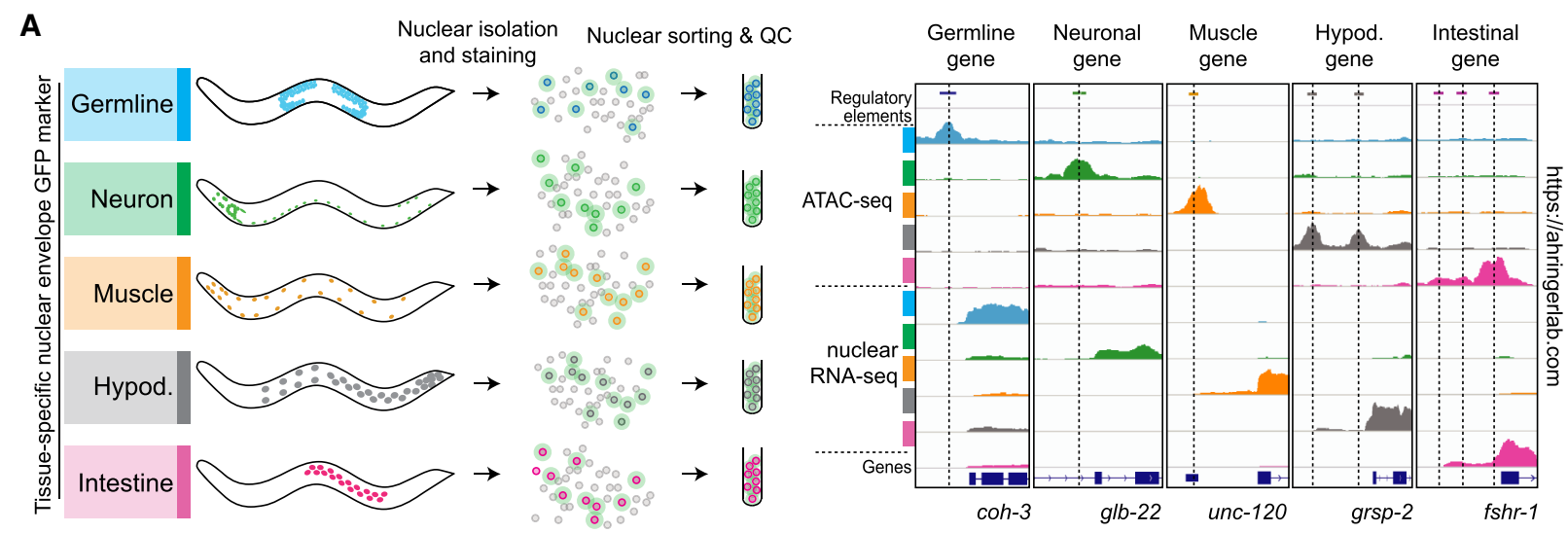

B

Accessible sites $(n=25,205)$

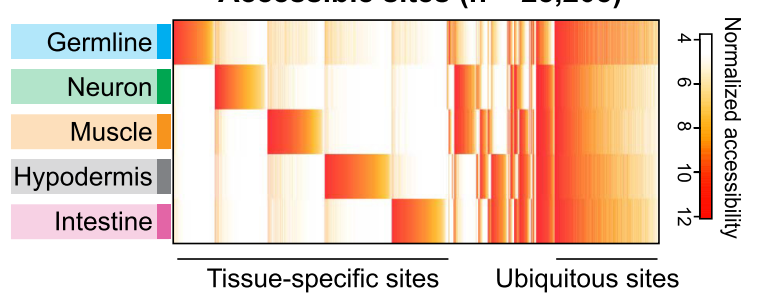

C
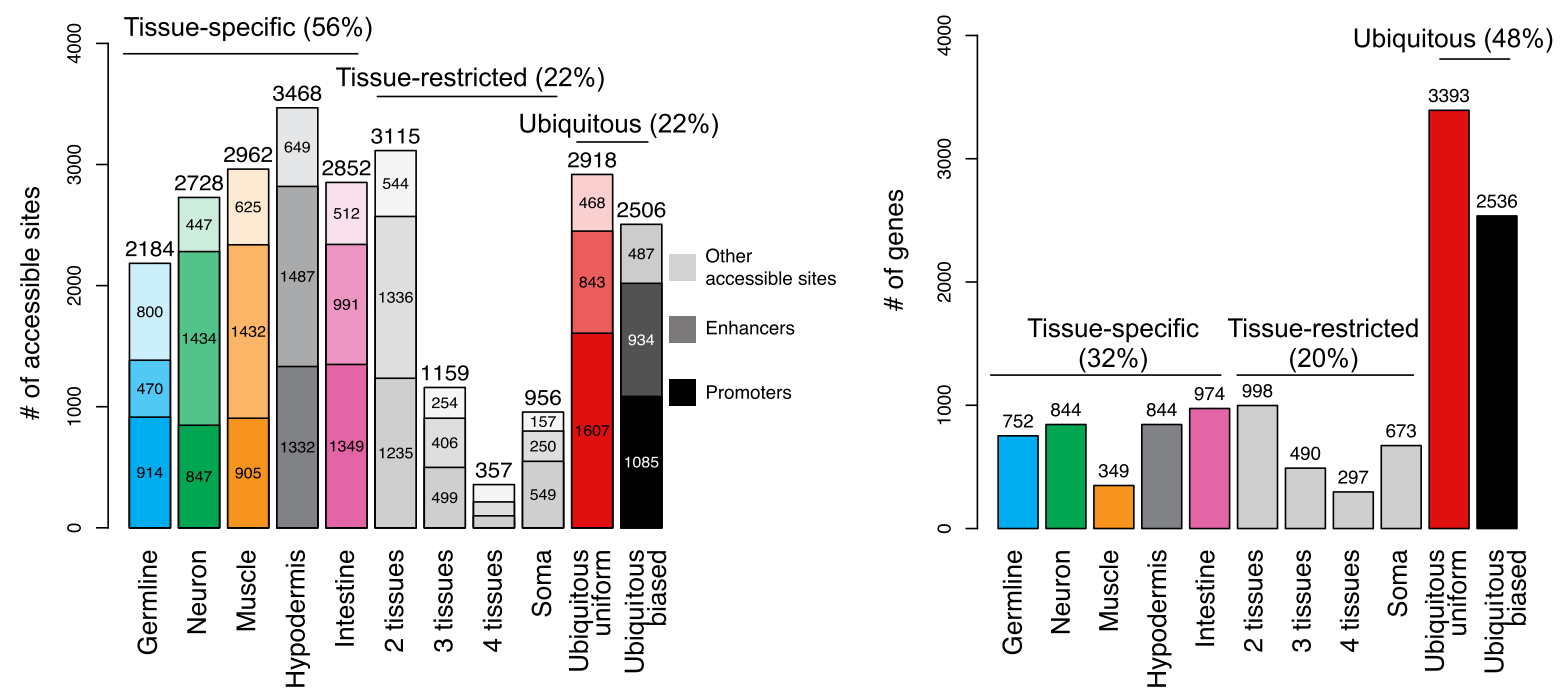

Figure 1. Tissue-specific profiling of chromatin accessibility and gene expression in C. elegans tissues. (A) Procedure to perform tissue-specific nuclear RNA-seq and ATAC-seq experiments. Representative results at known tissue-specific loci are shown on the right. (B, top) Heatmap of normalized accessibility $\left(\log _{2}\right.$ RPM) for 25,205 classified sites. (Bottom) Classification of the accessible sites into tissue-specific, tissue-restricted, or ubiquitous classes. Protein-coding promoters are in dark colors, enhancers are lighter, and other accessible sites (e.g., noncoding promoters, unassigned promoters, other elements) are lightest. (C, top) Heatmap of normalized gene expression ( $\log _{2}$ TPM) for 12,301 classified protein-coding genes. (Bottom) Classification of genes into tissuespecific, tissue-restricted, or ubiquitous classes. For classification procedure, see Methods. Unclassified sites and genes are not shown.

Table S1). The samples cover $\sim 90 \%$ of cells of adult worms but do not include the pharynx, glia, or somatic gonad.

We previously defined 42,245 accessible elements across C. elegans development and ageing in whole animals (Jänes et al. 2018). Because most genes in C. elegans are trans-spliced to a 22nt leader RNA (Blumenthal 2012), leading to removal and degradation of the initial $5^{\prime}$ sequence between the promoter and the trans-splice site, the beginning of the mature mRNA does not usu- ally correspond to the transcription initiation site. Therefore, nuclear RNA-seq patterns were used to annotate accessible sites identified using ATAC-seq (Jänes et al. 2018). Accessible elements were annotated as promoters based on the presence of nuclear transcription initiation signal within 125 bp downstream from the peak of accessibility and transcription elongation signal linking the element to a gene (unidirectional promoters) or genes (bidirectional promoters). Elements with transcription initiation 
signal but lacking transcription elongation signal were annotated as putative enhancers, and those lacking signals of transcriptional activity were classified as "other."

To classify the tissue specificity of gene expression and accessibility and to identify new elements, we performed ATAC-seq and nuclear RNA-seq on sorted nuclei from the five tissues. Biological replicates were highly concordant (Supplemental Fig. S2D,E), and known tissue-specific loci showed expected activities (Fig. 1A). By using the procedures by Jänes et al. (2018), we identified and annotated 5269 additional accessible elements through these new data, bringing the total to 47,514 . This added 2218 new putative enhancers (hereafter referred to as enhancers) and 901 new promoters; 11,806 protein-coding genes (58\%) have at least one high-confidence promoter.

Finally, we classified the tissue specificity of element accessibility and gene expression using a set of conservative rules (see Methods). Excluding elements and genes with low signal in all assessed samples, 25,205 (53\%) of the 47,514 accessible sites and $12,301(61 \%)$ of the 20,222 protein-coding genes were classified (Fig. 1B,C; Supplemental Table S2).

We observed that the chromatin accessibility of regulatory elements is largely tissue specific. The majority of regulatory elements (56\%) are accessible in only a single tissue, with the rest having tissue restricted (22\%) or ubiquitous accessibility (22\%); the latter were split into those with relatively uniform accessibility (less than threefold difference between any two tissues) and those with biased accessibility (Fig. 1B). For gene expression, the largest class of genes (48\%) had ubiquitous expression, with the remainder having tissue-specific (32\%) or tissue-restricted (20\%) expression (Fig. 1C). The gene expression classification showed good overlap with previously published annotations (Supplemental Fig S2G,H; Cao et al. 2017; Kaletsky et al. 2018). We observed that the nuclear RNA data sets have minor cross-contamination that appears, in part, to be bulk cytoplasmic RNA released during nuclear isolation, resulting in tissue-specific genes with high expression (e.g., muscle myosin gene unc-54) being classified as ubiquitous biased. Hereafter, when studying ubiquitous genes and elements, we specifically focus on the ubiquitous-uniform class and for simplicity refer to them as "ubiquitous."

The data provide a comprehensive view of chromatin accessibility and transcriptional landscapes in the five major C. elegans tissues. To facilitate access and analyses of these new tissue-specific and previous development data sets (Jänes et al. 2018), we created RegAtlas, a C. elegans regulatory atlas (https://ahringerlab.com/). Below, we analyze features of genes and regulatory elements active in different tissues.

\section{Germline-active and soma-restricted genes have distinctive regulatory architectures}

To investigate whether general rules could be discerned that govern different types of spatial expression patterns, we focused on genes with ubiquitous or tissue-specific expression and compared the number, type, and arrangement of regulatory elements associated with genes from each class. About $15 \%$ of C. elegans genes are organized in operons (Reinke and Cutter 2009), where two or more genes are initially transcribed into a single transcript that is separated by trans-splicing. Therefore, for analyses involving promoters, we only included nonoperon genes and first genes in operons. As reported previously (Reinke and Cutter 2009), we found that genes organized in operons preferentially have ubiquitous or germline-specific patterns of expression (Fig. 2A).
As expected, most (77\%) ubiquitously expressed genes with at least one classified promoter are associated with a ubiquitously active promoter (Supplemental Table S2). We observed that half (54\%) of the ubiquitous genes have just a single promoter, whereas $16 \%$ have a relatively complex regulatory architecture containing three or more promoters (Fig. 2B). To explore these differences, we separated ubiquitously expressed genes into groups based on promoter number (one, two, or three or more). We observed that single-promoter genes are enriched for functions in basic cellular processes, whereas those with three or more promoters are enriched for developmental functions (Fig. 2C). Multipromoter ubiquitous genes also have more enhancers than single-promoter genes, are more often controlled by unidirectional promoters, and have more and longer introns (Fig. 2D-G).

As for ubiquitous genes, tissue-specific genes are generally associated with one or more promoters specific for the corresponding tissue (78\%) (Fig. 2H). However, we observed that a group of genes with germline-specific expression have ubiquitously accessible promoters (Fig. 2H). We found that these genes are enriched for being targets of the repressive Rb/DREAM complex (13-fold enrichment, $P$-value $=5.10^{-13}$ ), and in line with this, they are enriched for cell cycle and cell division functions (Supplemental Fig S3A,B; Latorre et al. 2015; Goetsch et al. 2017). This suggests that the predominantly germline expression of these genes is achieved via their silencing in somatic tissues (Petrella et al. 2011; Wu et al. 2012).

By comparing the different tissue-specific classes, we found that germline-specific genes show extensive differences compared with somatic genes. First, germline-specific genes have fewer promoters and enhancers than somatic genes (Fig. 2B,D); 65\% of germline-specific genes with at least one classified promoter have a single promoter and no associated enhancer compared with $38 \%$ of somatic genes. The promoters of germline genes are more often bidirectional than those of somatic genes (Fig. 2E), and germline genes also have fewer and shorter introns, similar to ubiquitously expressed single-promoter genes (Fig. 2F,G).

A significant fraction of expressed genes with at least one annotated promoter (33\%) have more than one promoter, and alternative promoters are frequently active in the same tissue (Fig. 2I; Supplemental Fig S3C; Supplemental Table S2), suggesting that alternative promoters may play a role in the regulation of expression levels. To investigate this, we examined the relationship between the number of regulatory elements and gene expression level. Among ubiquitously expressed genes, we found that the number of promoters and enhancers was positively correlated with gene expression (Fig. 2J). Similarly, tissue-specific genes with two tissue-specific promoters have higher gene expression levels than those with only one (Fig. $2 \mathrm{~K}$ ). We also note that $15 \%$ of the ubiquitously expressed genes with two promoters have one tissue-specific promoter in addition to a ubiquitously active one, which could be a mechanism to increase gene expression specifically in a particular tissue (Supplemental Fig. S3D). These results suggest that an important but often overlooked role of regulatory elements is to augment gene expression rather than being necessary for its expression per se. This could explain some cases in which deletion of an individual regulatory element does not have an obvious effect on gene expression, despite the regulatory element having transcriptional activity in transgenic assays (Dukler et al. 2017; Catarino and Stark 2018).

To summarize, we found that the regulatory architecture of genes is related to their function and expression pattern (Fig. 2L). Ubiquitous genes required for fundamental cellular

\section{Genome Research}

www.genome.org 


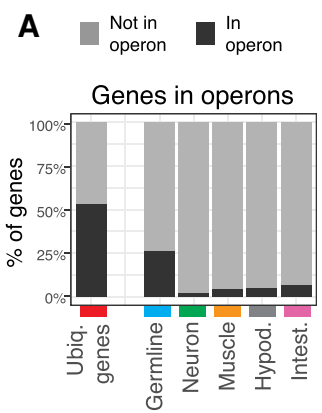

C

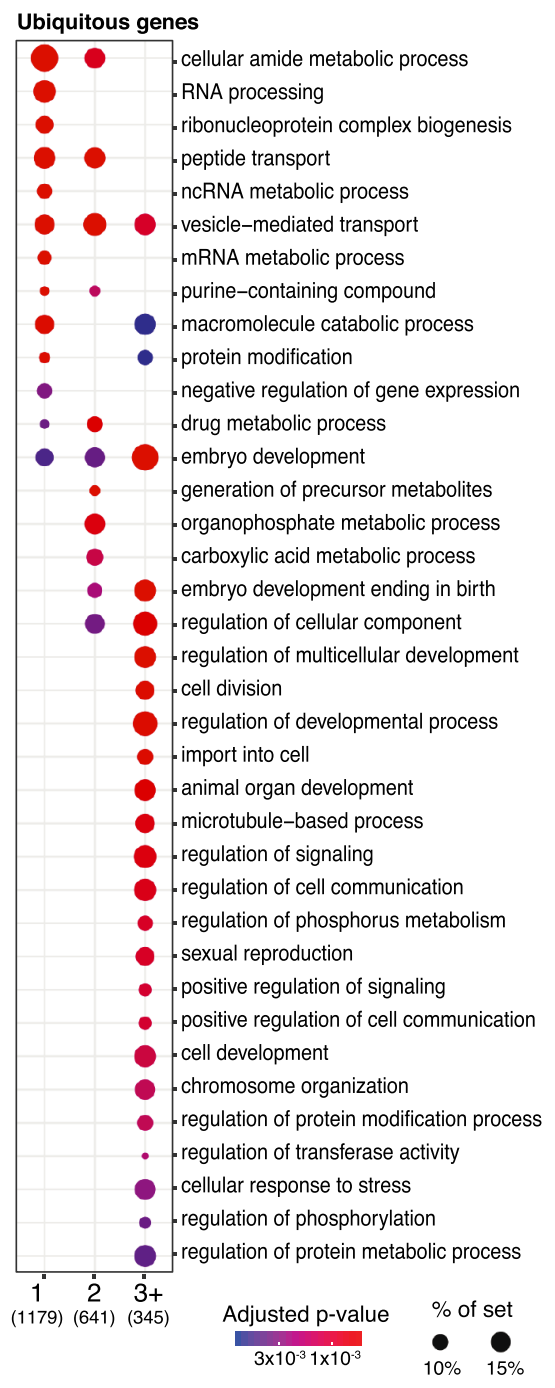

D

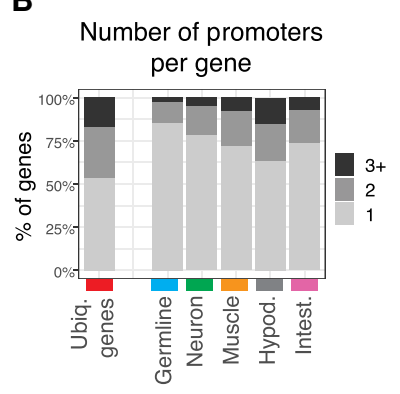

F

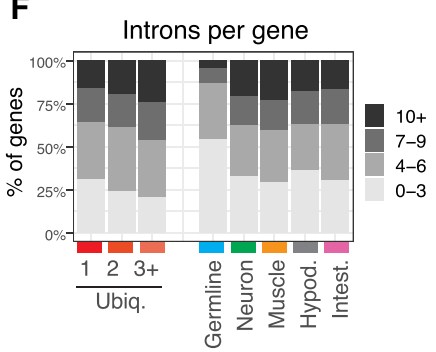

H

H Gene classes

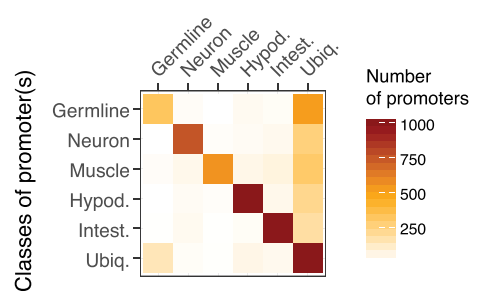

J
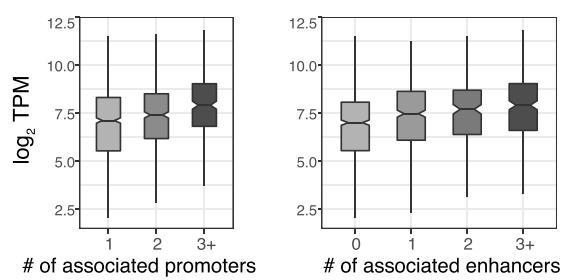

L

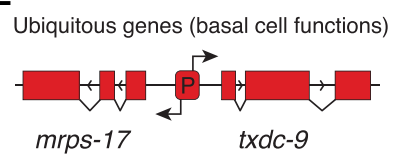

Germline-specific genes

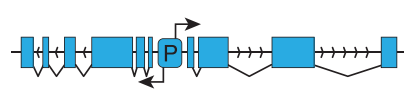

snpc-3.1

puf-7
E

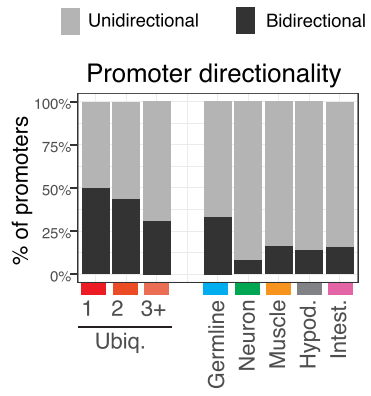

G

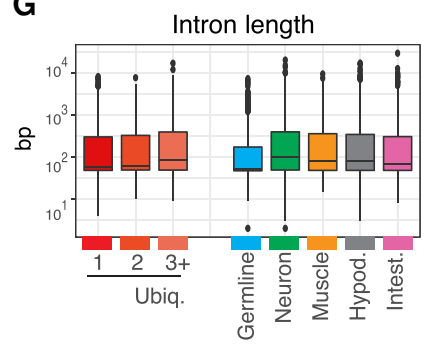

I

Promoter classes

for 2-promoter genes

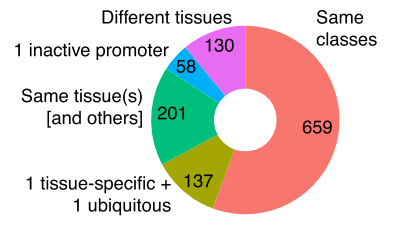

K Tissue-specific gene expression

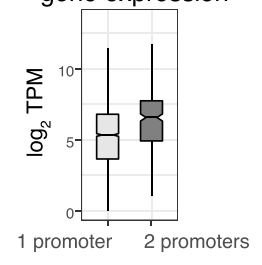

Figure 2. Regulatory architectures of ubiquitous, germline, and soma-restricted genes have distinctive features. $(A)$ Percentage of genes organized in an operon for each gene class. (B) Percentage of genes with one, two, or three or more promoters for each gene class. (C) GO terms from Biological Process ontology enriched in ubiquitous genes with one, two, or three or more annotated promoters. $(D)$ Percentage of genes with zero, one, two, or three or more enhancers associated with genes of each expression class. Only genes with at least one annotated promoter are considered. (E) Percentage of unidirectional or bidirectional protein-coding promoters for each gene class. ( $F)$ Percentage of genes with the indicated number of introns for each gene class. $(G)$ Intron length for each gene class. $(H)$ Classes of promoters associated with genes of each expression class. Only the major promoter classes are displayed. For all results, see Supplemental Table S2. (I) Concordance of promoter classes for genes with two promoters. ( $)$ Gene expression levels in whole young adults for ubiquitous genes with one, two, or three or more promoters (left) or with zero, one, two, or three or more enhancers (right). ( $K$ ) Gene expression levels of tissue-specific genes with one promoter or two promoters specifically active in the same tissue. In panels $B$ through $K$, only first genes in operons and nonoperon genes were considered. ( $L$, left) Examples of the simple regulatory architecture shared by ubiquitous genes and germline-specific genes. (Right) Examples of more complex architectures found at developmental ubiquitous genes (e.g., lin-45) or somatic tissue-specific genes (e.g., mlt-10). 
processes and germline-specific genes tend to have a simple architecture consisting of a single promoter that is often bidirectional. In contrast, ubiquitous genes with functions associated with multicellular life often have a more complex architecture of multiple regulatory elements that can have diverse tissue specificity. Somatic tissue-specific genes usually have one or more regulatory elements accessible only in the matching tissue. Finally, the positive relationship between gene expression and the number of regulatory elements supports a role in modulating the level of gene expression.

\section{Ubiquitous and germline-specific promoters have a stereotypical architecture with well-positioned nucleosomes}

The tissue-specific differences in gene regulatory architectures prompted us to investigate whether differences also occurred at the level of promoters. By comparing accessibility patterns of different classes of promoters, we observed that germline-specific and ubiquitously active promoters were flanked by regions of increased accessibility and associated with more nucleosome-sized ATAC-seq fragments, suggesting the presence of well-positioned nucleosomes (Supplemental Fig. S4A,B). The flanking ATAC-seq signal at germline promoters was also present in proliferative stages (L1 and L3 larval stages), indicating that it is not simply a characteristic of germline nuclei undergoing meiosis (Supplemental Fig. S4C).

To investigate this potential signature of positioned nucleosomes, we used ATAC-seq fragment density plots (also known as "V-plots") (Henikoff et al. 2011) to visualize the distribution of fragment lengths relative to the distance to the promoter center. Over promoters flanked by positioned -1 and +1 nucleosomes, V-plots show stereotypical patterns with a central concentration of small fragments at the nucleosome-depleted region (NDR) and larger fragments over $+1 /-1$ nucleosomes on either side of the NDR (Fig. 3A; Henikoff et al. 2011). In line with this, a signature of -1 and +1 nucleosomes is readily apparent at ubiquitous promoters in all tissues, as well as at germline-specific promoters (Fig. 3B). However, somatic tissue-specific promoters lack this signature of well-positioned $+1 /-1$ nucleosomes (Fig. 3B; Supplemental Fig. S5A,B), regardless of the level of expression of the associated gene (Supplemental Fig. S5C) or promoter directionality (Supplemental Fig. S5D,E).

To explore this further, we used the ATAC-seq data to compute nucleosome occupancy probability profiles as previously described (Schep et al. 2015). This revealed a high probability of +1 and -1 nucleosome occupancy at consistent positions relative to transcription start sites (TSSs) of ubiquitous and germline-specific promoters (Fig. 3C; Supplemental Fig. S4D). In contrast, somatic tissue-specific promoters were characterized by lower -1 and +1 nucleosome occupancy and a larger range of nucleosome positions relative to TSSs (Fig. 3C; Supplemental Fig. S4D). We found that the $5^{\prime}$ edges of +1 nucleosomes at ubiquitous and germline-specific promoters have narrow distributions relative to TSSs, with median distances of $22 \mathrm{bp}$ for ubiquitous promoters and $12 \mathrm{bp}$ for germline-specific promoters. In contrast, +1 nucleosomes at somatic tissue-specific promoters have much wider distributions and larger median distances (Fig. 3C,D). We also observed that NDR widths are smaller, and divergent promoter TSSs are closer together for ubiquitous and germline-specific promoters compared with somatic tissue-specific promoters (Fig. 3D; Supplemental Fig. $\mathrm{S} 4 \mathrm{D})$. Of note, the median NDR widths at ubiquitous and germ- line-specific promoters are $140 \mathrm{bp}$ and $125 \mathrm{bp}$, which would be too short to accommodate a nucleosome.

To identify sequence features that may be responsible for these differences, we performed motif analyses. We observed that ubiquitous and germline-specific promoters share a T-rich motif with 10-bp spacing that was not present at somatic tissuespecific promoters (Fig. 4A). Previous studies mostly performed in vitro or in yeast have implicated 10-bp WW $(\mathrm{W}=\mathrm{A} / \mathrm{T})$ periodicity in nucleosome positioning and observed SS periodicity in antiphase with WW (Satchwell et al. 1986; Ioshikhes et al. 1996; Wang and Widom 2005; Johnson et al. 2006; Segal et al. 2006; Field et al. 2008; Mavrich et al. 2008a; Struhl and Segal 2013).

To investigate whether the T-rich motif we identified was part of a larger WW periodic signal involved in +1 nucleosome positioning at ubiquitous and germline-specific promoters in C. elegans, we measured WW dinucleotide periodicity from $-50 \mathrm{bp}$ to +300 bp relative to TSSs. We observed that ubiquitous and germline-specific promoter regions harbor a strong 10-bp periodic WW signal that extends for $>150 \mathrm{bp}$ and that the periodicity signal coincides with +1 nucleosome position (Fig. 4B,D; Supplemental Fig. S6A). Furthermore, at these promoters we found that 10-bp WW periodicity strength is correlated with +1 nucleosome occupancy (Fig. 4E). In contrast, the 10-bp periodic WW signal was not detected at somatic tissue-specific promoters, in line with the absence of positioned +1 nucleosomes at these promoters (Fig. 4B,C; Supplemental Fig. S6A,B). Therefore, an extended 10bp periodic WW signal specific to ubiquitously active and germline active promoters is associated with nucleosome position and occupancy.

By examining the contribution of different dinucleotides to the WW signal, we found that TT periodicity peaks in the $5^{\prime}$ region of the +1 nucleosome of ubiquitous and germline-specific promoters, $\sim 50 \mathrm{bp}$ downstream from the TSS, and makes a larger contribution than other dinucleotides (Fig. 4C,D; Supplemental Fig. S6A). A weaker AA periodic signal peaks at the $3^{\prime}$ edge of the nucleosome (Fig. 4C,D; Supplemental Fig. S6A), and AT and TA dinucleotides do not show any robust periodic signal (Supplemental Fig. S6A). We note that a 10-bp SS periodicity antiphase with WW is also present at ubiquitous and germline-specific promoters (Supplemental Fig. S6A,B).

The strength of the 10-bp periodic WW signal is similar at +1 nucleosomes of bidirectional and unidirectional ubiquitous promoters (Supplemental Fig. S6C). Periodicity is also present at -1 nucleosomes of the unidirectional promoters, although the signal is weaker (Supplemental Fig. S6C). We also note that WW periodicity strength differs among ubiquitous promoters of ubiquitous genes. WW periodicity is stronger at single-promoter genes, which are enriched for basal cell functions, compared with ubiquitous promoters of genes with three or more promoters, which are enriched for developmental functions (Fig. 2C; Supplemental Fig. S6C).

We next investigated the tissue specificity and position of other promoter elements. The Inr initiator sequence, the Sp1 motif, and the TATA-box are three well-known core promoter elements that have been previously observed in C. elegans promoters (Saito et al. 2013; Chen et al. 2013). Inr motifs were detected in all promoter classes; however, somatic tissue-specific promoters showed higher enrichment than ubiquitous and germlinespecific promoters (Supplemental Fig. S7; Supplemental Table S3). We further observed that the Sp1 and TATA box motifs were both predominantly associated with somatic tissue-specific promoters, with large and unexpected tissue biases (Supplemental Fig. S7;

\section{Genome Research}

www.genome.org 
A

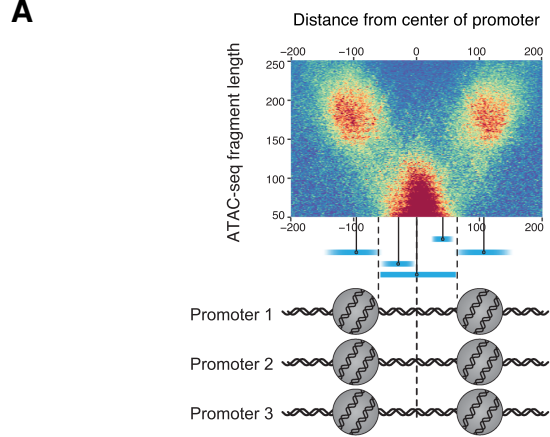

B

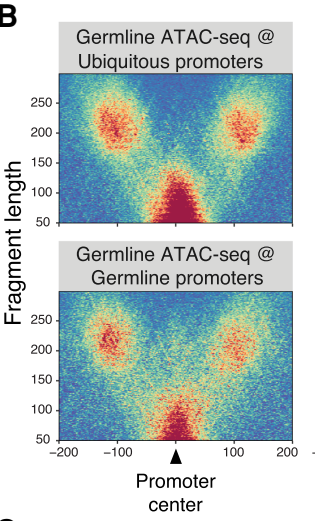

C

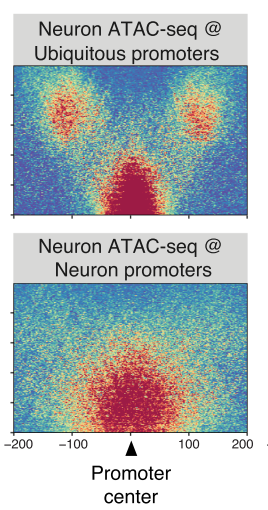

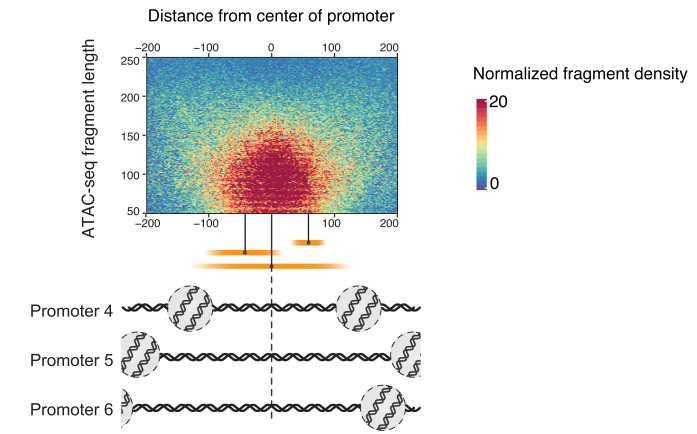
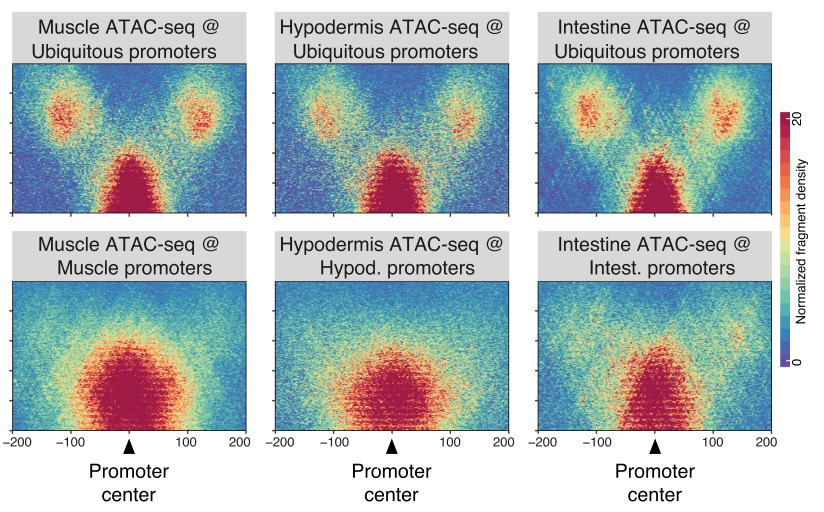

Probability of nucleosome occupancy
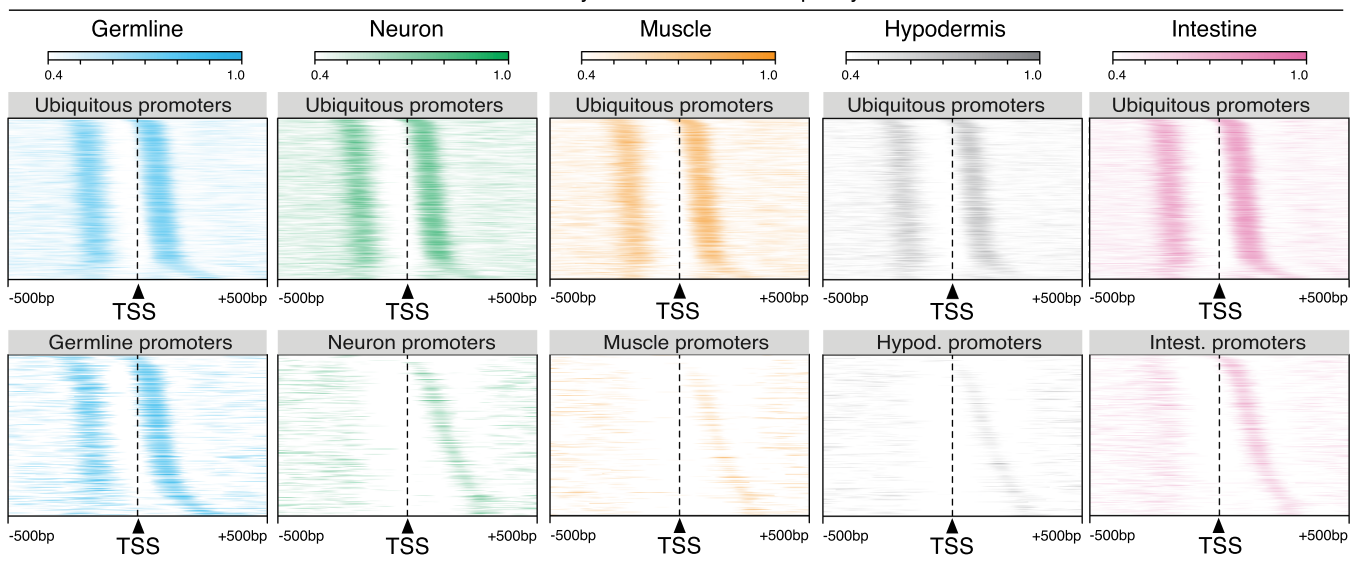

D
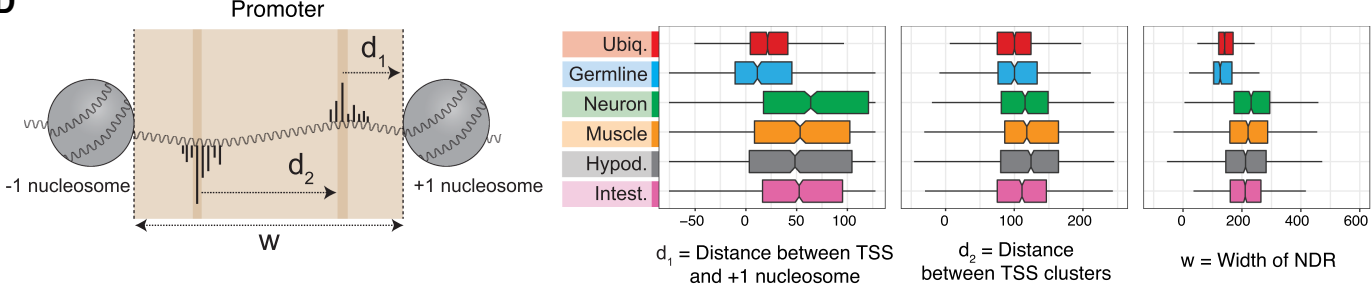

Figure 3. Ubiquitous and germline-specific promoters have a stereotypical architecture with well-positioned nucleosomes. (A) Interpretation of two ATAC-seq fragment density plots (also known as "V-plots"). The dense cluster of short fragments at the promoter centers represents the nucleosome-depleted region (NDR), whereas the dense clusters of longer fragments located -100 and +100 bp away from the promoter centers are indicative of aligned $-1 /+1$ flanking nucleosomes. (B) ATAC-seq fragment density plots (V-plots) over different classes of promoters. The $x$-axis represents the distance between the fragment midpoint and the promoter center. The $y$-axis represents ATAC-seq fragment length. The color scale indicates the normalized density of ATAC-seq fragments. (C) Tissue-specific nucleosome occupancy probability over different classes of promoters aligned at their TSS. Only promoters with experimentally defined forward and reverse TSSs are considered. Rows are ordered by the distance between TSS and +1 nucleosome. ( $D$, left) Schematic of the distance metrics measured in promoters: $\left(d_{1}\right)$ distance between the mode TSS and the +1 nucleosome edge; $\left(d_{2}\right)$ distance between modes of divergent TSSs within the same promoter; $(w)$ width of the NDR. (Right) $d_{1}, d_{2}$, and $w$ distance metrics for different classes of promoters. The metrics for ubiquitous promoters were measured using nucleosome occupancy probability track derived from whole young adult ATAC-seq data (Jänes et al. 2018). 

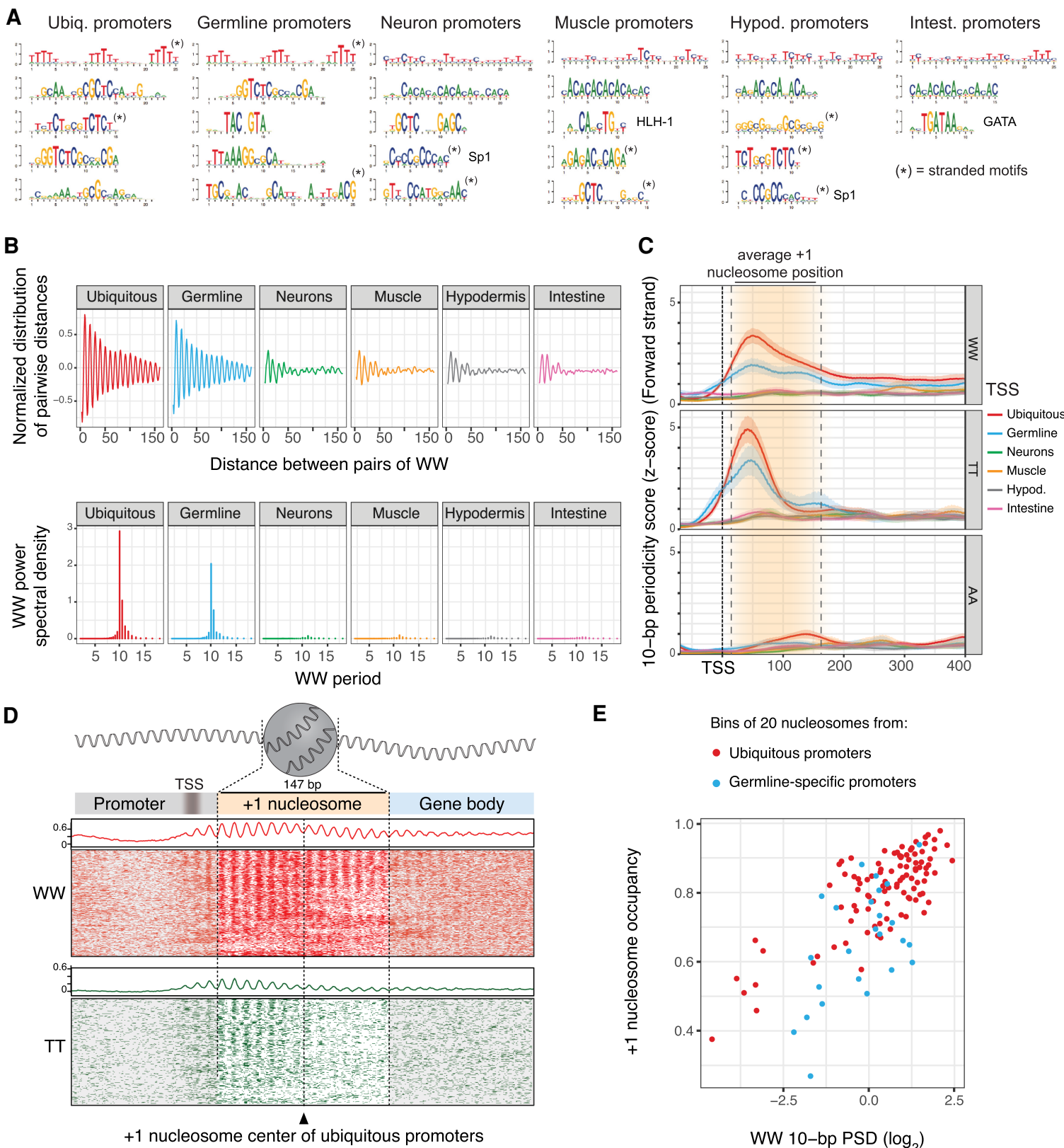

E
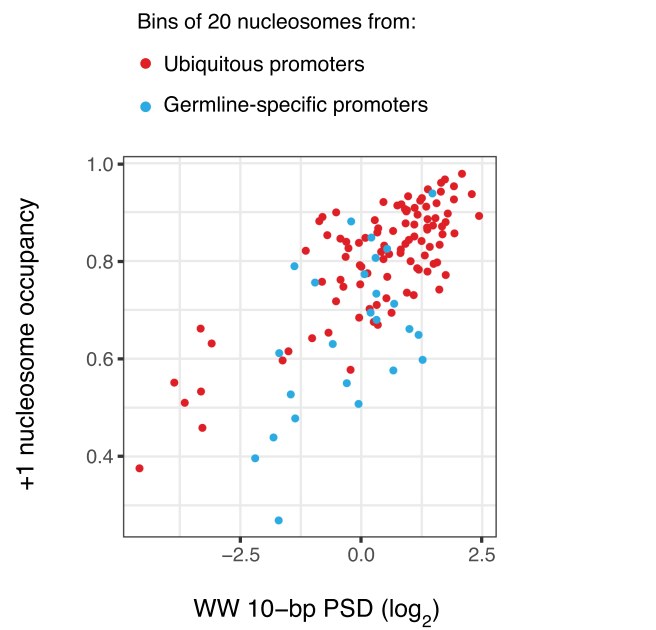

Figure 4. Ubiquitous and germline-specific promoters have strong 10-bp WW periodicity correlated with nucleosomes. (A) Motifs enriched in different classes of promoters. Sequences from -75 to $+105 \mathrm{bp}$ around the promoter centers were considered. $(B$, top $)$ Normalized distribution of pairwise distances between WW dinucleotides found in the sequences from $-50 \mathrm{bp}$ to +300 bp relative to TSSs, for different classes of promoters. (Bottom) Associated WW power spectral densities (PSDs). (C) Metaplots of WW, TT, and AA 10-bp periodicity scores at different classes of promoters, aligned at TSSs. The +1 nucleosome position observed at ubiquitous and germline promoters ( $20-167$ bp downstream from the TSS) is displayed by the shaded orange area delimited by dotted lines. $(D) W W($ red) and TT (green) dinucleotide occurrences observed at +1 nucleosomes of ubiquitous promoters (400-bp window centered at nucleosome dyads). Rows were shifted up to $5 \mathrm{bp}$ to highlight the phased 10-bp periodic patterns. Summed dinucleotide occurrences are represented on top of each heatmap by a line plot. The average TSS positions of ubiquitous promoters ( $20 \mathrm{bp}$ upstream of the +1 nucleosome edge) are displayed by the shaded gray area. (E) Correlation between +1 nucleosome occupancy and 10-bp WW periodicity in ubiquitous and germline-specific promoters. The +1 nucleosomes were binned by their nucleosome occupancy score, and the overall 10-bp WW periodicity was assessed in each bin (approximately 20200 -bp long nucleosomal sequences centered at nucleosome dyads). The $y$-axis represents the average nucleosome occupancy in each bin.

Supplemental Table S3). The Sp1 motif, peaking at $-45 \mathrm{bp}$ from the TSS, is enriched at neural, muscle, and hypodermal promoters but not at intestinal promoters, whereas the TATA-box motif was predominantly found at hypodermal and intestinal promoters. We also observed that somatic tissue-specific promoters share repeated dinucleotide composition biases not found in ubiquitous or germline-specific promoters (Fig. 4A; Supplemental Fig. S7; Supplemental Table S3).

The de novo motif analyses also uncovered motifs associated with promoters active in single tissues (Fig. 4A; Supplemental Fig. S7; Supplemental Table S3). For example, as expected, many intestinal promoters harbor a GATA motif, whereas the HLH-1 motif is

\section{Genome Research}

www.genome.org 
found specifically at muscle promoters (Supplemental Fig. S7; Chen et al. 1994; McGhee et al. 2007). These motifs and others have peak positions within the NDR, often $\sim 45$ bp upstream of the TSS (Supplemental Fig. S7). Thus, there are tissue-specific differences in both core promoter elements and TF binding motifs.

In summary, our results uncover two largely different types of promoter architecture. Ubiquitous and germline-specific promoters have well-positioned $+1 /-1$ nucleosomes that are highly associated with a periodic 10-bp WW signal and stereotypically positioned with the $5^{\prime}$ edge $\sim 20$ bp downstream from the TSS, and they have relatively short NDRs. In contrast, +1 nucleosomes of somatic tissue-specific promoters have low occupancy and inconsistent positioning relative to TSSs, and NDRs are wider. In addition, core promoter and transcription factor motifs show strong tissue biases.

\section{Ten-base-pair WW periodicity at ubiquitous promoters is a} feature of nonmammalian genomes

We next asked whether a 10-bp periodic WW signal is a feature associated with +1 nucleosomes of ubiquitous promoters of other animals. Ten-base-pair periodic WW sequences have been observed at +1 nucleosomes in yeast, Drosophila, and zebrafish but not in mammals (Albert et al. 2007; Mavrich et al. 2008a,b; Tolstorukov et al. 2009; Ioshikhes et al. 2011; Haberle et al.
2014; Wright and Cui 2019). However, whether 10-bp WW periodicity is associated with promoters of particular types has not been investigated.

We first examined TSS sets that represent all genes in Drosophila, zebrafish, mouse, and human (see Methods). As expected, we detected 10-bp WW periodicity signals downstream from Drosophila and zebrafish TSSs, but not human TSSs, and we found that this signal was also not detected in mouse (Fig. 5A). As in C. elegans, we observed that the WW periodicity signals in Drosophila and zebrafish peaked in the $5^{\prime}$ half of +1 nucleosomes (Fig. $5 \mathrm{~A}$ ).

We then investigated subsets of promoters to ask whether 10bp WW periodicity signals are associated with ubiquitously active promoters, and to compare with signals at promoters with regulated activity. By using the coefficient of variation of gene expression $(\mathrm{CV})$ as a metric, we considered genes in the bottom $20 \%$ of $\mathrm{CV}$ values to have broad ubiquitous expression and those in the top 20\% to have highly regulated expression (e.g., tissue specificity). As found in C. elegans, we observed that promoters of broadly expressed genes in Drosophila and zebrafish have higher 10-bp WW periodicity signals than those of highly regulated genes. In contrast, neither the broadly active nor the regulated groups of mouse and human promoters had detectable WW periodicity signals (Fig. $5 \mathrm{~B}, \mathrm{C})$. These results suggest that 10 -bp WW periodicity signals are a conserved feature of ubiquitously active promoters in nonmammalian animals.

A
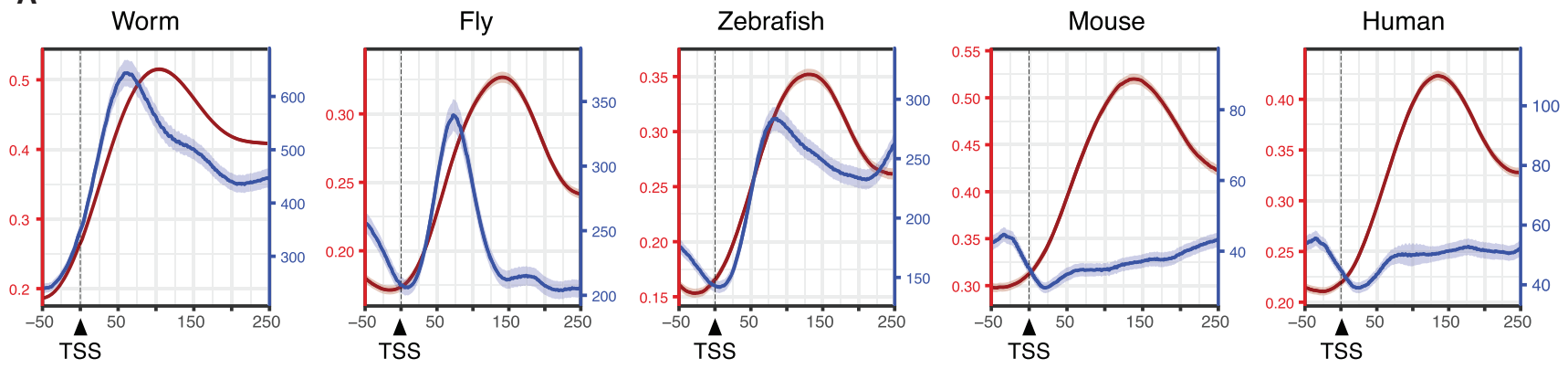

Nucleosome occupancy

WW 10-bp periodicity

B

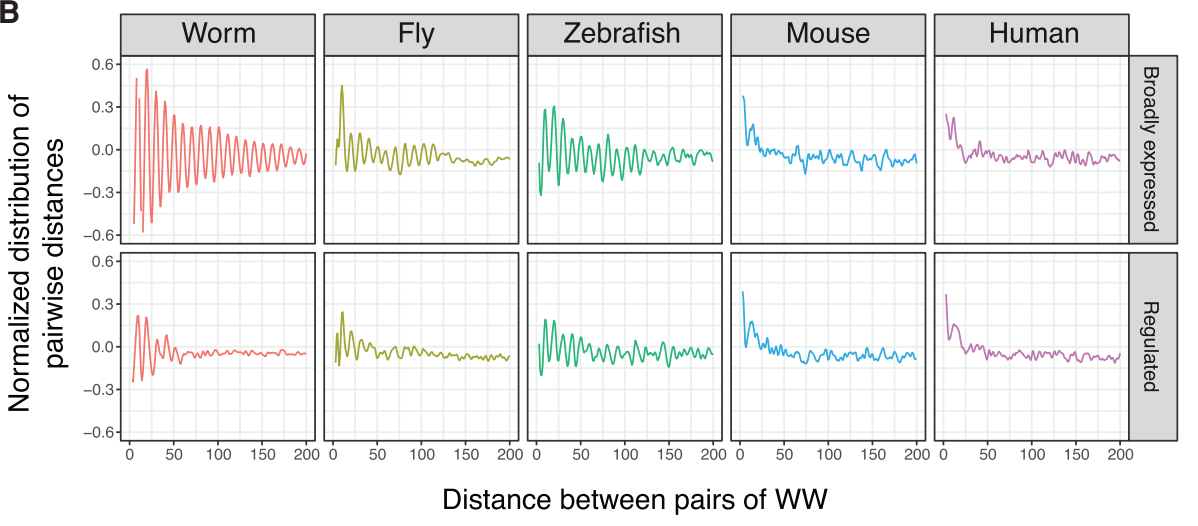

C

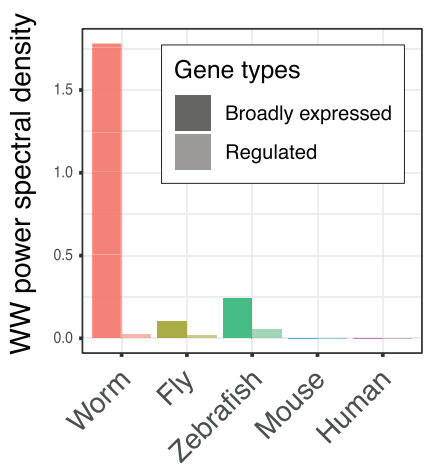

Figure 5. Ten-base-pair WW periodicity at ubiquitous promoters is a feature of nonmammalian genomes. ( $A$ ) Nucleosome occupancy probability scores (red; left axis) and 10-bp WW periodicity (blue; right axis) at worm, fly, zebrafish, mouse, and human TSSs. (B) Normalized distribution of pairwise distances between WW dinucleotides found in the sequences from $-50 \mathrm{bp}$ to $+300 \mathrm{bp}$ relative to TSSs, for genes with broad expression (top row; $20 \%$ lowest gene expression CV scores) or regulated expression (bottom row; $20 \%$ highest gene expression CV scores) in worm, fly, zebrafish, mice, and human. (C) Associated WW power spectral density values at a 10-bp period. 


\section{Discussion}

Determining the regulatory architectures that drive different gene expression patterns is necessary for understanding how the genome encodes development. Through comprehensive analyses of gene expression and chromatin accessibility in five C. elegans tissues covering $\sim 90 \%$ of cells, we show that most genes have either ubiquitous or tissue-specific expression, and we describe extensive differences between their regulatory architectures. The expression of ubiquitous genes involved in basic biological processes as well as that of germline-specific genes is often controlled by single promoters, whereas soma-specific and ubiquitous genes involved in developmental processes have more alternative promoters and enhancers.

We also found that the majority of regulatory elements have tissue-specific accessibility, and we identified differences in sequence composition between promoters active in different tissues. We found that a strong +1 nucleosome position coinciding with a 10 -bp periodic WW signal is a key feature of ubiquitous and germline-specific promoters in C. elegans. The association of 10-bp WW periodicity and nucleosome rotational position was first noted by Travers and colleagues in chicken and is thought to aid nucleosome positioning by conferring sequence-dependent bendability to the DNA polymer (Zhurkin et al. 1979; Trifonov 1980; Drew and Travers 1985). Such periodicity has been observed in nucleosomal sequences in different eukaryotes, including C. elegans, but its specific association with different gene types was unknown (Satchwell et al. 1986; Ioshikhes et al. 1996, 2011; Widom 2001; Johnson et al. 2006; Segal et al. 2006; Peckham et al. 2007; Field et al. 2008; Mavrich et al. 2008a,b; Struhl and Segal 2013; Forrest et al. 2014; Haberle et al. 2014; Dreos et al. 2016; Pich et al. 2018). We note that 10-bp periodic An/Tn-clusters (PATCs) have also been shown to be associated with introns of C. elegans germline expressed genes located in repressive chromatin domains and to help facilitate their transcription (Fire et al. 2006; FrøkjærJensen et al. 2016). The mechanism of this regulation is unclear, but the 10-bp periodic $\mathrm{A} / \mathrm{T}$ signals suggest that nucleosome positioning may play a role.

In contrast to ubiquitous and germline-specific promoters, +1 nucleosomes of somatic tissue-specific promoters are not associated with a 10-bp WW periodicity signal, have lower occupancy, and have inconsistent position relative to the TSS. Instead, we observed strong biases in the enrichment of core motifs at these promoters. TATA boxes are primarily found in hypodermal and intestinal promoters, whereas Sp1 motifs are most highly enriched in neuronal promoters. In addition, tissue-specific motifs are present, and these often have peak positions around $-50 \mathrm{bp}$ relative to the mode TSS.

Structural studies of the preinitiation complex (PIC) showed that it covers the region from about $-45 \mathrm{bp}$ to +20 bp relative to the TSS (Louder et al. 2016; Robinson et al. 2016; Schilbach et al. 2017). The $5^{\prime}$ edge of +1 nucleosomes at $C$. elegans ubiquitous and germline promoters are located $\sim 20$ bp downstream from the TSS, which would be at the $3^{\prime}$ edge of the PIC. This supports the model initially proposed in yeast whereby a positioned $+1 \mathrm{nu}$ cleosome could facilitate PIC complex assembly by interacting with TFIID (Fig. 6A,B; Jiang and Pugh 2009). At soma-specific promoters, which lack strongly positioned nucleosomes, the binding of core or tissue-specific TFs $\sim 45$ bp upstream of the TSS might help to locally recruit and/or to position the PIC (Fig. 6A,B). These models are not mutually exclusive, and additional mechanisms also contribute to promoter activity.

\section{A Ubiquitous and germline-specific promoters}

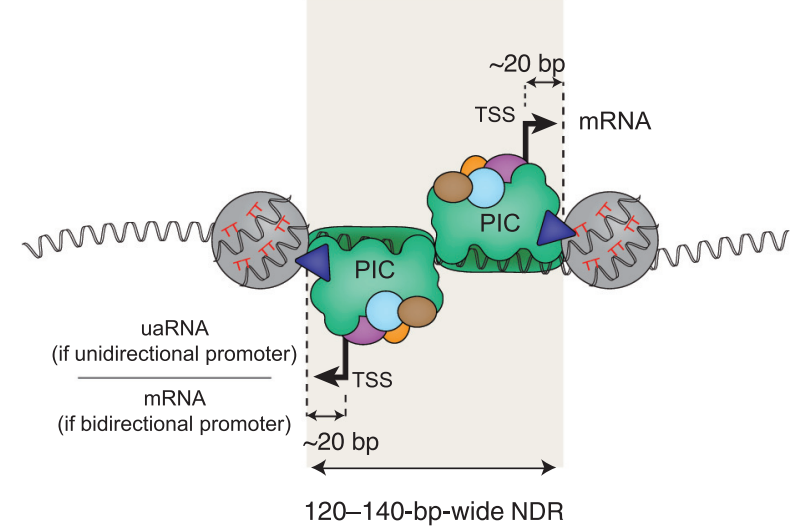

B

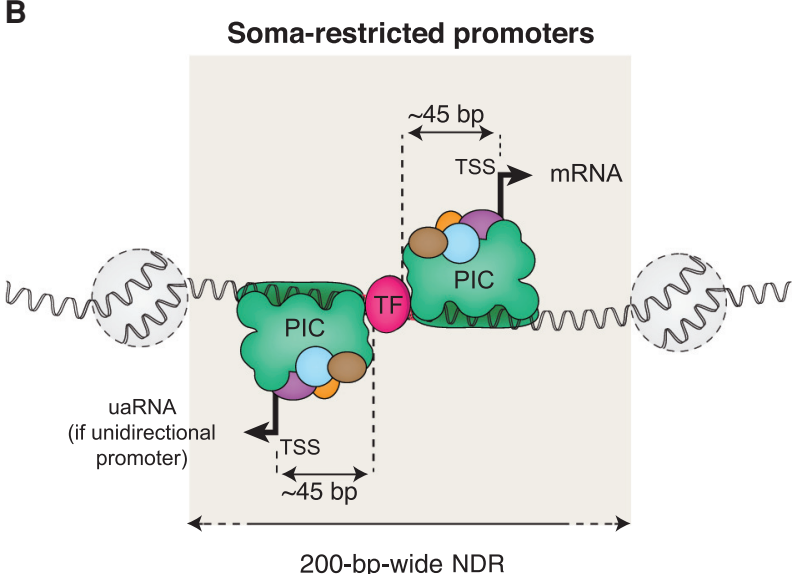

Figure 6. Two models of preinitiation complex (PIC) positioning at promoters. The nucleosome organization and sequences features found in ubiquitous, germline-specific, and somatic tissue-specific promoters suggest that two models of PIC recruitment exist. $(A)$ In ubiquitous and germline-specific promoters (i.e., germline-active promoters), nucleosomes flank a narrow 120- to 140-bp-wide NDR. Positioning of these nucleosomes is facilitated by the underlying DNA sequence, which harbors highly periodic WW (mainly TT) dinucleotides. Thus, the PIC assembling at the NDR is physically constrained by the +1 nucleosome edge, resulting in transcription initiation $\sim 20$ bp upstream of the +1 nucleosome edge. Many of these promoters lead to bidirectional elongative transcription. Otherwise, upstream-antisense RNA (uaRNA) are transcribed. (B) In soma-restricted promoters, NDRs are wider (>200 bp), and flanking nucleosomes are weakly positioned and not reproducibly aligned relative to the TSS. Core and transcription factors recruited to the NDR facilitate assembly and positioning of the PIC, resulting in transcription initiation -45 to -50 bp downstream.

Similar to C. elegans, we observed that a 10-bp WW periodicity signal is also associated with promoter +1 nucleosomes of broadly expressed genes in zebrafish and Drosophila. This is consistent with a previously described enrichment of 10-bp periodicity in AA and TT dinucleotides downstream from zygotic TSSs in zebrafish (Haberle et al. 2014). A weak genome-wide AA/TT periodicity was previously noted in Drosophila but not associated with any gene feature (Mavrich et al. 2008b). In contrast, the periodic WW signal is not detected at promoters of broadly expressed genes in mouse and human, despite their having well-positioned +1 nucleosomes. This is consistent with reports showing a low 10-bp WW periodicity in mammal genomes, either around TSSs

\section{Genome Research}

www.genome.org 
(Tolstorukov et al. 2009; Wright and Cui 2019) or genome-wide (Pich et al. 2018). Multiple factors have been shown to contribute to nucleosome positioning in eukaryotes, including intrinsic DNA sequence, chromatin remodelers, DNA-binding proteins, and RNA polymerase machinery (Jiang and Pugh 2009; Struhl and Segal 2013). We suggest that 10-bp WW periodicity is an ancient conserved signal that contributes to +1 nucleosome positioning at ubiquitously active promoters of nonmammalian eukaryotes, especially those of genes with basal cell functions, whereas nucleosome positioning in mammals may rely on other mechanisms (Struhl and Segal 2013).

In addition to illuminating understanding of regulatory architectures, we provide extensive data sets and annotation of gene expression and accessible chromatin across tissues, available at the C. elegans regulatory atlas (RegAtlas) (Supplemental Fig. S8; https://ahringerlab.com/RegAtlas/). These data and tools will be key resources that facilitate future studies of C. elegans gene expression regulation by the scientific community.

\section{Methods}

\section{Nuclear sorting}

Animals were obtained by growing synchronized starved L1 larvae at $25 \mathrm{C}$ in standard S-basal medium with HB101 bacteria for 40-42 $\mathrm{h}$, resulting in collections predominantly containing late L4s and young adults with no embryos. After sucrose flotation and washing in M9 buffer, worms were frozen into "popcorn" by dripping concentrated slurry into liquid nitrogen. Nuclei were isolated as previously detailed (Jänes et al. 2018), with minor modifications. Approximately 20,000 to 200,000 frozen worms were broken by smashing using a BioPulverizer, and then the frozen powder was thawed in $8 \mathrm{~mL}$ egg buffer (25 mM HEPES at pH 7.3, $118 \mathrm{mM}$ $\mathrm{NaCl}, 48 \mathrm{mM} \mathrm{KCl}, 2 \mathrm{mM} \mathrm{CaCl} 2,2 \mathrm{mM} \mathrm{MgCl}_{2}$ ). Broken worms were pelleted by spinning at $800 \mathrm{~g}$ for $3 \mathrm{~min}$ and then resuspended in $8 \mathrm{~mL}$ of buffer $\mathrm{A}(0.3 \mathrm{M}$ sucrose, $10 \mathrm{mM}$ Tris at $\mathrm{pH} 7.5,10 \mathrm{mM}$ $\mathrm{MgCl}_{2}, 1 \mathrm{mM}$ DTT, $0.5 \mathrm{mM}$ spermidine, $0.15 \mathrm{mM}$ spermine, protease inhibitors [Roche complete, EDTA free], and 0.025\% IGEPAL CA-630). The sample was dounced (two strokes) in a 14-mL stainless steel tissue grinder (VWR) and then spun at $100 \mathrm{~g}$ for $6 \mathrm{~min}$ to pellet remaining worm fragments. The supernatant was kept (nuclei batch 1) and the pellet resuspended in a further $7 \mathrm{~mL}$ of buffer A and dounced for 30 strokes. This was spun at $100 \mathrm{~g}$ for $6 \mathrm{~min}$ to pellet debris, and the supernatant was kept (nuclei batch 2). The first fraction was enriched for germline nuclei, whereas the second fraction was enriched for somatic nuclei. Nuclei quality was assessed by microscopy.

Following isolation, nuclei were immunostained by adding phycoerythrin-coupled anti-GFP antibody (Biolegend 338003) at 1:200 in $7 \mathrm{~mL}$ of buffer $\mathrm{A}$, and 280 units of murine RNase inhibitor (M0314S) were added to protect RNA from being degraded. Nuclei were kept slowly rotating at $4^{\circ} \mathrm{C}$ in the dark for $1-16 \mathrm{~h}$. Debris was removed by spinning at $100 \mathrm{~g}$ for $6 \mathrm{~min}$ at $4^{\circ} \mathrm{C}$, and then nuclei were pelleted $\left(2000 \mathrm{~g}\right.$ for $20 \mathrm{~min}$ at $4^{\circ} \mathrm{C}$ ), washed in $6 \mathrm{~mL}$ of buffer $\mathrm{A}$, and resuspended in buffer A containing $80 \mathrm{U} / \mathrm{mL}$ murine RNase inhibitor at a concentration of approximately 10-15 million nuclei per milliliter. Finally, nuclei were filtered on a $30-\mu \mathrm{m}$ mesh (CellTrics 04-0042-2316) and stained with $0.025 \mu \mathrm{g} / \mathrm{mL}$ DAPI. Nuclei quality was assessed immediately before sorting by microscopy.

Nuclear sorting was performed at $4^{\circ} \mathrm{C}$ using a Sony SH800Z sorter fitted with a 100- $\mu \mathrm{m}$ sorting chip and auto-calibrated. Nuclei were gated using the DAPI signal, and PE-positive nuclei were gated using PE-H/BSC-A signal. DAPI gating depended on which nuclei were being sorted (e.g., intestine nuclei are $32 \mathrm{~N}$ ). A recording speed of more than 15,000 nuclei per second ensured a sorting efficiency $>80 \%$. Nuclei were sorted into $15-\mathrm{mL}$ Falcon tubes containing $500 \mu \mathrm{L}$ of buffer A with $800 \mathrm{U} / \mathrm{mL}$ murine RNase inhibitor. Nuclei were sorted in batches of 1 million and then processed for downstream applications. The purity and integrity of each batch of nuclei were assessed by recording an aliquot of sorted nuclei in a second pass in the sorter and by microscopy. All sorted samples used in this study had a purity $>95 \%$. Sorted nuclei were intact, as revealed by the circular DAPI signal observed, as well as the GFP signal outlining the nuclear envelope (see Supplemental Fig. S2B).

\section{ATAC-seq}

One million sorted nuclei were pelleted $\left(2000 \mathrm{~g}\right.$ for $20 \mathrm{~min}$ at $4^{\circ} \mathrm{C}$ ) and resuspended in $1 \times$ Tn5 buffer $(10 \mathrm{mM}$ Tris at $\mathrm{pH} 8,5 \mathrm{mM}$ $\mathrm{MgCl}_{2}, 10 \% \mathrm{DMF}$ ) at a final concentration of about 500,000 nuclei per milliliter; $2.5 \mu \mathrm{L}$ of Tn5 (Illumina FC-121-1030) was added to $47.5 \mu \mathrm{L}$ (about 25,000 nuclei) of the suspension. ATAC-seq was then performed as previously described (Jänes et al. 2018). ATAC-seq libraries were generated from two biological replicates for each tissue and were sequenced in both single-end and paired-end modes. Single ATAC-seq libraries were made for L1 and L3 muscle (SE-sequenced) and L3 germline (PE-sequenced). PGC-specific ATAC-seq data at the L1 stage were obtained from Lee et al. (2017).

\section{RNA-seq}

RNA was extracted from 1 million sorted and washed nuclei using the standard procedure (Jänes et al. 2018). A minimum of $20 \mathrm{ng}$ of total nuclear RNA was used to make long nuclear RNA-seq libraries. Long nuclear RNA (>200 nt) was isolated using Zymo Clean and Concentrate columns (R1013); rRNA was removed using the Ribo-Zero rRNA removal kit (MRZH11124); and stranded libraries were prepared with the NEBNext ultra directional RNA library prep kit (E7420S). Long nuclear RNA-seq libraries were generated from two biological replicates for each tissue and were sequenced in paired-end mode. We observed that all tissue-specific libraries have noticeable background for abundant tissue-specific mRNAs (e.g., muscle myosin unc-54). This appears to be due at least in part by contamination by whole-animal cytoplasmic RNA released during nuclear isolation, as the RNA in the unexpected tissue is predominantly spliced.

\section{Data processing}

Data were processed as previously described (Jänes et al. 2018) and aligned to WBcel235/ce11 genome. Further details are given in the Supplemental Methods.

To assess the reproducibility of biological replicate data sets, we used site accessibility or gene expression values to compute pairwise Euclidean distances between each data set and pairwise Pearson's correlation scores. ATAC-seq and RNA-seq biological replicates showed high concordance (Supplemental Fig. S2D).

\section{Classification of accessible sites}

First, accessibility at each site in each sample was calculated as reads per million (RPM) values. RPMs of biological replicates were averaged to obtain a single accessibility score for each site in each tissue. Sites with accessibility $<8$ RPM in every tissue were not further studied.

Then, estimation of accessibility fold-changes (FCs) and adjusted $P$-values were computed between all pairs of tissues using 
the DESeq2 package (Love et al. 2014). A site was considered significantly differentially accessible (DA) between two tissues if there was a FC greater than three and an adjusted $P$-value $<0.01$. A FC of three between consecutive tissues was used as a threshold to determine the tissue specificity of accessible sites. Classification details are provided in the Supplemental Material.

\section{Classification of genes}

Long nuclear RNA-seq stranded fragments were assigned to C. elegans gene annotations (WBCel235, release 92) using the featureCounts program with "-t gene -s 2 -Q 10 -p" options. Estimation of expression FCs and adjusted $P$-values were computed between pairs of tissues using the DESeq2 package. A gene was considered significantly differentially expressed (DE) between two tissues if there was a FC greater than three and an adjusted $P$-value $<0.01$.

In each sample, gene expression was calculated as transcripts per million (TPM) values. TPMs of biological duplicates were then averaged to obtain a single gene expression value for each tissue.

The rules used to classify accessible sites were also used to classify genes, with a detection threshold of 5 TPM. A small number of germline-specific genes (151) with maximal expression in L4 (Jänes et al. 2018) were classified as sperm specific and not included in this study.

\section{Gene Ontology (GO) analysis}

GO enrichment analyses were performed using the gProfileR 0.6.7 package (Reimand et al. 2007), filtering for redundant GO terms using the hier_filtering = moderate option. To compare GO enrichment across several groups, the clusterProfiler 3.10.1 package (Yu et al. 2012) was used, filtering for redundant terms using REVIGO (Supek et al. 2011). Only GO terms with Bonferroni-adjusted $P$-values lower than 0.05 were kept.

\section{ATAC-seq fragment density plots}

ATAC-seq fragment density plots, also known as V-plots (Henikoff et al. 2011), were generated using the VplotR package (release 0.4.0; https://github.com/js2264/VplotR). Flanking nucleosome enrichment scores were calculated from the V-plots as illustrated in Supplemental Figure S5A.

\section{Nucleosome occupancy tracks and +1 nucleosome mapping}

Processed BAM files from paired-end ATAC-seq duplicates of each tissue or from whole organism young adults (Jänes et al. 2018) were merged. For each class of promoter (germline, neuron, muscle, hypodermis, intestine, and ubiquitous promoters), the nucleoATAC Python package (Schep et al. 2015) was used to compute the probability of nucleosome occupancy from $-1 \mathrm{~kb}$ to $+1 \mathrm{~kb}$ from promoter centers in each tissue (germline, neuron, muscle, hypodermis, intestine, and whole organism).

Putative +1 and -1 nucleosome positions were determined for each set of tissue-specific promoters using the corresponding tissue-specific nucleosome occupancy probability track and for ubiquitous promoters using the whole-organism nucleosome occupancy probability track (Jänes et al. 2018). We assigned the center of the putative +1 nucleosome to the local maximum of the nucleosome occupancy probability within $200 \mathrm{bp}$ downstream from the forward TSS mode. Similarly, the center of the -1 nucleosome summit was assigned to the local maximum of the occupancy probability within $200 \mathrm{bp}$ upstream of the reverse TSS mode. Only coding promoters with experimentally determined forward and reverse TSSs were considered.

\section{Motif identification and enrichment analyses}

Motifs enriched in different sets of promoters ( $-75 \mathrm{bp}$ to $+105 \mathrm{bp}$ from promoter centers) were identified using MEME in stranded mode and a zero-order background model (-markov_order 0). MEME mode was set to "any number of repetitions" (-mod anr), and motif widths were restricted to $6-25 \mathrm{bp}$. The five motifs found most enriched in each tissue (with an $E$-value threshold of 0.05 ) were retrieved. Unstranded motifs (found twice as complementary sequences, because MEME was run in stranded mode) were manually combined. PWMs for the Initiator (Inr) and the TATA motif were obtained from Jin et al. (2006). Motif mapping to promoters was performed in R v 3.5.2 (R Core Team 2019) using the Biostrings 2.50.2 package, the GenomicRanges 1.34 .0 package, and the TFBSTools 1.20.0 package with a relScore threshold set to 0.8 .

\section{Dinucleotide periodicity}

To estimate dinucleotide periodicity in sets of sequences (e.g., -50 to +300 bp sequences around ubiquitous, germline, or somatic tissue-specific TSSs in Fig. 4B, or -50 to +300 bp sequences around TSSs from different organisms in Fig. 5A), the getPeriodicity() function from the periodicDNA 0.2.0 package was used with default parameters. Briefly, the distribution of distances between all possible pairs of dinucleotides in the set of sequences was computed and corrected for distance decay and smoothed by a moving average window of three, and power spectral densities were retrieved by applying a fast Fourier transform to the normalized distribution.

To generate 10-bp dinucleotide periodicity score tracks, the generatePeriodicityTrack() function from the periodicDNA package (release 0.2.0, https://github.com/js2264/periodicDNA) was used with default parameters. Briefly, a running 10-bp dinucleotide periodicity score was calculated by applying a fast Fourier transform (stats 3.5.2 package) on the distribution of distances between pairs of dinucleotides (e.g., WW......WW) found in 100-bp long sequences (2-bp increments).

\section{Phasing of nucleosomal sequences}

To observe the 10-bp periodic occurrence of a dinucleotide in putative +1 nucleosomes, sequences ( $400 \mathrm{bp}$ centered at the nucleosome dyads) were first clustered by $k$-means based on the dinucleotide occurrences in each sequence, and then the clusters were rephased within a -/+5-bp range using the lag value estimated by the $\operatorname{ccf}()$ function from the stats 3.5.2 package.

\section{Sets of annotations in fly, fish, mouse, and human}

In worms, experimentally annotated TSSs were used (Jänes et al. 2018). In fly and zebrafish (respectively dm6 and danRer10 genome versions), TSSs were assigned to the first base of the genes using TxDb.Dmelanogaster.UCSC.dm6.ensGene 3.4 .4 and TxDb.Drerio.UCSC.danRer10.refGene 3.4.4 gene models with the GenomicFeatures 1.34.7 package in R. In mouse and human, FANTOM CAGE data sets (Lizio et al. 2015) were used to retrieve the dominant TSS closest to the gene annotation. CV values were retrieved from Gerstein et al. (2014) for worm, fly, and human or computed using gene expression data sets from Pervouchine et al. (2015) for mouse and White et al. (2017) for zebrafish. Genes with the $20 \%$ lowest CVs were considered broadly expressed, and those with the $20 \%$ highest CVs were considered regulated.

\section{Nucleosome occupancy in fly, fish, mouse, and human}

Nucleosome occupancy tracks were generated as described for worms using nucleoATAC with the following ATAC-seq data sets:

\section{Genome Research}

www.genome.org 
SRR6171265 (Haines and Eisen 2018) in fly, SRR5398228 (Quillien et al. 2017) in zebrafish, SRR5470874 (Benchetrit et al. 2019) in mouse, and SRR891268 (Buenrostro et al. 2013) in human.

\section{Software availability}

Processed data and all annotations are available and can be either dynamically explored or anonymously downloaded at https:// ahringerlab.com/RegAtlas/. Software for V-plotting and for analysis of periodicity of DNA motifs developed for this study are available as $\mathrm{R}$ packages. VplotR release 0.4.0 (Source code VplotR0.4.0.tar.gz) is available at https:// github.com/js2264/VplotR/ releases/tag/v0.4.0 and periodic DNA release 0.2.0 (Source code periodicDNA-0.2.0.tar.gz) is available at https://github.com/ js2264/periodicDNA/releases/tag/v0.2.0 (both are also available as Supplemental Code).

\section{Data access}

All raw and processed sequencing data generated in this study have been submitted to the NCBI Gene Expression Omnibus (GEO; https://www.ncbi.nlm.nih.gov/geo/) under accession number GSE141213.

\section{Competing interest statement}

The authors declare no competing interests.

\section{Acknowledgments}

We thank all the members of the Ahringer laboratory and particularly F. Carelli and A. Frapporti for fruitful discussions and comments on the manuscript. We thank A. Appert and K. Harnish for support in sequencing. The work was supported by a Wellcome Trust Senior Research Fellowship to J.A. (101863) and a Medical Research Council DTP studentship to J.S. We also acknowledge core support from the Wellcome Trust (092096) and Cancer Research UK (C6946/A14492).

Author contributions: J.S. and J.A. conceived and designed the studies. J.S. performed experiments unless otherwise noted. J.S., M.C., and C.C. generated the reporter strains. J.S. and Y.D. prepared RNA-seq libraries. J.S. and J.J. processed the data. J.S. analyzed the data. J.S. and J.A. wrote the manuscript. J.A. supervised the study and provided funding.

\section{References}

Albert I, Mavrich TN, Tomsho LP, Qi J, Zanton SJ, Schuster SC, Pugh BF. 2007. Translational and rotational settings of H2A.Z nucleosomes across the Saccharomyces cerevisiae genome. Nature 446: 572-576. doi:10.1038/ nature05632

Andersson R, Sandelin A. 2019. Determinants of enhancer and promoter activities of regulatory elements. Nat Rev Genet 21: 71-87. doi:10.1038/ s41576-019-0173-8

Bahr C, von Paleske L, Uslu VV, Remeseiro S, Takayama N, Ng SW, Murison A, Langenfeld K, Petretich M, Scognamiglio R, et al. 2018. A Myc enhancer cluster regulates normal and leukaemic haematopoietic stem cell hierarchies. Nature 553: 515-520. doi:10.1038/nature25193

Benchetrit H, Jaber M, Zayat V, Sebban S, Pushett A, Makedonski K, Zakheim Z, Radwan A, Maoz N, Lasry R, et al. 2019. Direct induction of the three pre-implantation blastocyst cell types from fibroblasts. Cell Stem Cell 24: 983-994.e7. doi:10.1016/j.stem.2019.03.018

Blumenthal T. 2012. Trans-splicing and operons in C. elegans. WormBook 111. doi:10.1895/wormbook.1.5.2

Buenrostro JD, Giresi PG, Zaba LC, Chang HY, Greenleaf WJ. 2013. Transposition of native chromatin for fast and sensitive epigenomic profiling of open chromatin, DNA-binding proteins and nucleosome position. Nat Methods 10: 1213-1218. doi:10.1038/nmeth.2688
Cao J, Packer JS, Ramani V, Cusanovich DA, Huynh C, Daza R, Qiu X, Lee C, Furlan SN, Steemers FJ, et al. 2017. Comprehensive single-cell transcriptional profiling of a multicellular organism. Science 357: 661-667. doi:10.1126/science.aam8940

Catarino RR, Stark A. 2018. Assessing sufficiency and necessity of enhancer activities for gene expression and the mechanisms of transcription activation. Genes Dev 32: 202-223. doi:10.1101/gad.310367.117

The $C$. elegans Sequencing Consortium. 1998. Genome sequence of the nematode C. elegans: a platform for investigating biology. Science 282: 2012-2018. doi:10.1126/science.282.5396.2012

Chen L, Krause M, Sepanski M, Fire A. 1994. The Caenorhabditis elegans MYOD homologue HLH-1 is essential for proper muscle function and complete morphogenesis. Development 120: 1631-1641.

Chen RA-J, Down TA, Stempor P, Chen QB, Egelhofer TA, Hillier LW, Jeffers TE, Ahringer J. 2013. The landscape of RNA polymerase II transcription initiation in C. elegans reveals promoter and enhancer architectures. Genome Res 23: 1339-1347. doi:10.1101/gr.153668.112

Cusanovich DA, Reddington JP, Garfield DA, Daza RM, Aghamirzaie D, Marco-Ferreres R, Pliner HA, Christiansen L, Qiu X, Steemers FJ, et al. 2018. The cis-regulatory dynamics of embryonic development at single-cell resolution. Nature 555: 538-542. doi:10.1038/nature25981

Davuluri RV, Suzuki Y, Sugano S, Plass C, Huang TH-M. 2008. The functional consequences of alternative promoter use in mammalian genomes. Trends Genet 24: 167-177. doi:10.1016/j.tig.2008.01.008

Dreos R, Ambrosini G, Bucher P. 2016. Influence of rotational nucleosome positioning on transcription start site selection in animal promoters. PLoS Comput Biol 12: e1005144. doi:10.1371/journal.pcbi.1005144

Drew HR, Travers AA. 1985. DNA bending and its relation to nucleosome positioning. J Mol Biol 186: 773-790. doi:10.1016/0022-2836(85) 90396-1

Dukler N, Gulko B, Huang Y-F, Siepel A. 2017. Is a super-enhancer greater than the sum of its parts? Nat Genet 49: 2-3. doi:10.1038/ng.3759

Ernst J, Kheradpour P, Mikkelsen TS, Shoresh N, Ward LD, Epstein CB, Zhang $\mathrm{X}$, Wang $\mathrm{L}$, Issner $\mathrm{R}$, Coyne $\mathrm{M}$, et al. 2011. Mapping and analysis of chromatin state dynamics in nine human cell types. Nature 473: 4349. doi:10.1038/nature09906

Field Y, Kaplan N, Fondufe-Mittendorf Y, Moore IK, Sharon E, Lubling Y, Widom J, Segal E. 2008. Distinct modes of regulation by chromatin encoded through nucleosome positioning signals. PLoS Comput Biol 4: e1000216. doi:10.1371/journal.pcbi.1000216

Fire AZ, Alcazar R, Tan F. 2006. Unusual DNA structures associated with germline genetic activity in Caenorhabditis elegans. Genetics 173: 1259-1273. doi:10.1534/genetics.106.057364

Forrest ARR, Kawaji H, Rehli M, Baillie JK, de Hoon MJL, Haberle V, Lassmann T, Kulakovskiy IV, Lizio M, Itoh M, et al. 2014. A promoterlevel mammalian expression atlas. Nature 507: 462-470. doi:10.1038/ nature13182

Frøkjær-Jensen C, Jain N, Hansen L, Davis MW, Li Y, Zhao D, Rebora K, Millet JRM, Liu X, Kim SK, et al. 2016. An abundant class of non-coding DNA can prevent stochastic gene silencing in the C. elegans germline. Cell 166: 343-357. doi:10.1016/j.cell.2016.05.072

Gerstein MB, Lu ZJ, Van Nostrand EL, Cheng C, Arshinoff BI, Liu T, Yip KY, Robilotto R, Rechtsteiner A, Ikegami K, et al. 2010. Integrative analysis of the Caenorhabditis elegans genome by the modENCODE project. Science 330: 1775-1787. doi:10.1126/science.1196914

Gerstein MB, Rozowsky J, Yan K-K, Wang D, Cheng C, Brown JB, Davis CA, Hillier L, Sisu C, Li JJ, et al. 2014. Comparative analysis of the transcriptome across distant species. Nature 512: 445-448. doi:10.1038/ nature 13424

Goetsch PD, Garrigues JM, Strome S. 2017. Loss of the Caenorhabditis elegans pocket protein LIN-35 reveals MuvB's innate function as the repressor of DREAM target genes. PLoS Genet 13: e1007088. doi:10.1371/journal .pgen.1007088

Guerrero L, Marco-Ferreres R, Serrano AL, Arredondo JJ, Cervera M. 2010. Secondary enhancers synergise with primary enhancers to guarantee fine-tuned muscle gene expression. Dev Biol 337: 16-28. doi:10.1016/j .ydbio.2009.10.006

Haberle V, Lenhard B. 2016. Promoter architectures and developmental gene regulation. Semin Cell Dev Biol 57: 11-23. doi:10.1016/j.semcdb .2016.01.014

Haberle V, Li N, Hadzhiev Y, Plessy C, Previti C, Nepal C, Gehrig J, Dong X, Akalin A, Suzuki AM, et al. 2014. Two independent transcription initiation codes overlap on vertebrate core promoters. Nature 507: 381-385. doi:10.1038/nature12974

Haines JE, Eisen MB. 2018. Patterns of chromatin accessibility along the anterior-posterior axis in the early Drosophila embryo. PLoS Genet 14: e1007367. doi:10.1371/journal.pgen.1007367

Heinz S, Romanoski CE, Benner C, Glass CK. 2015. The selection and function of cell type-specific enhancers. Nat Rev Mol Cell Biol 16: 144-154. doi:10.1038/nrm3949 
Henikoff JG, Belsky JA, Krassovsky K, MacAlpine DM, Henikoff S. 2011. Epigenome characterization at single base-pair resolution. Proc Nat Acad Sci 108: 18318-18323. doi:10.1073/pnas.1110731108

Herr W. 1993. The SV40 enhancer: transcriptional regulation through a hierarchy of combinatorial interactions. Semin Virol 4: 3-13. doi:10.1016/ 1044-5773(93)80003-7

Ho JWK, Jung YL, Liu T, Alver BH, Lee S, Ikegami K, Sohn K-A, Minoda A, Tolstorukov MY, Appert A, et al. 2014. Comparative analysis of metazoan chromatin organization. Nature 512: 449-452. doi:10.1038/ nature13415

Ioshikhes I, Bolshoy A, Derenshteyn K, Borodovsky M, Trifonov EN. 1996. Nucleosome DNA sequence pattern revealed by multiple alignment of experimentally mapped sequences. J Mol Biol 262: 129-139. doi:10 $.1006 /$ jmbi.1996.0503

Ioshikhes I, Hosid S, Pugh BF. 2011. Variety of genomic DNA patterns for nucleosome positioning. Genome Res 21: 1863-1871. doi:10.1101/gr .116228 .110

Jänes J, Dong Y, Schoof M, Serizay J, Appert A, Cerrato C, Woodbury C, Chen R, Gemma C, Huang N, et al. 2018. Chromatin accessibility dynamics across C. elegans development and ageing. eLife 7: e37344. doi:10.7554/eLife. 37344

Jiang C, Pugh BF. 2009. Nucleosome positioning and gene regulation: advances through genomics. Nat Rev Genet 10: 161-172. doi:10.1038/ $\operatorname{nrg} 2522$

Jin VX, Singer GAC, Agosto-Pérez FJ, Liyanarachchi S, Davuluri RV. 2006. Genome-wide analysis of core promoter elements from conserved human and mouse orthologous pairs. BMC Bioinformatics 7: 114. doi:10 1186/1471-2105-7-114

Johnson SM, Tan FJ, McCullough HL, Riordan DP, Fire AZ. 2006. Flexibility and constraint in the nucleosome core landscape of Caenorhabditis elegans chromatin. Genome Res 16: 1505-1516. doi:10.1101/gr.5560806

Kaletsky R, Yao V, Williams A, Runnels AM, Tadych A, Zhou S, Troyanskaya OG, Murphy CT. 2018. Transcriptome analysis of adult Caenorhabditis elegans cells reveals tissue-specific gene and isoform expression. PLoS Genet 14: e1007559. doi:10.1371/journal.pgen.1007559

Kudron MM, Victorsen A, Gevirtzman L, Hillier LW, Fisher WW, Vafeados D, Kirkey M, Hammonds AS, Gersch J, Ammouri H, et al. 2018. The ModERN resource: genome-wide binding profiles for hundreds of Drosophila and Caenorhabditis elegans transcription factors. Genetics 208: 937-949. doi:10.1534/genetics.117.300657

Latorre I, Chesney MA, Garrigues JM, Stempor P, Appert A, Francesconi M, Strome S, Ahringer J. 2015. The DREAM complex promotes gene body H2A.Z for target repression. Genes Dev 29: 495-500. doi:10.1101/gad .255810 .114

Lee C-YS, Lu T, Seydoux G. 2017. Nanos promotes epigenetic reprograming of the germline by down-regulation of the THAP transcription factor LIN-15B. eLife 6: e30201. doi:10.7554/eLife.30201

Lenhard B, Sandelin A, Carninci P. 2012. Metazoan promoters: emerging characteristics and insights into transcriptional regulation. Nat Rev Genet 13: 233-245. doi:10.1038/nrg3163

Levine M. 2010. Transcriptional enhancers in animal development and evolution. Curr Biol 20: R754-R763. doi:10.1016/j.cub.2010.06.070

Liu C, Wang M, Wei X, Wu L, Xu J, Dai X, Xia J, Cheng M, Yuan Y, Zhang P, et al. 2019. An ATAC-seq atlas of chromatin accessibility in mouse tissues. Sci Data 6: 65. doi:10.1038/s41597-019-0071-0

Lizio M, Harshbarger J, Shimoji H, Severin J, Kasukawa T, Sahin S, Abugessaisa I, Fukuda S, Hori F, Ishikawa-Kato S, et al. 2015. Gateways to the FANTOM5 promoter level mammalian expression atlas. Genome Biol 16: 22. doi:10.1186/s13059-014-0560-6

Louder RK, He Y, López-Blanco JR, Fang J, Chacón P, Nogales E. 2016. Structure of promoter-bound TFIID and model of human pre-initiation complex assembly. Nature 531: 604-609. doi:10.1038/nature17394

Love MI, Huber W, Anders S. 2014. Moderated estimation of fold change and dispersion for RNA-seq data with DESeq2. Genome Biol 15: 550. doi:10.1186/s13059-014-0550-8

Lupiáñez DG, Spielmann M, Mundlos S. 2016. Breaking TADs: how alterations of chromatin domains result in disease. Trends Genet 32: 225237. doi:10.1016/j.tig.2016.01.003

Mavrich TN, Ioshikhes IP, Venters BJ, Jiang C, Tomsho LP, Qi J, Schuster SC Albert I, Pugh BF. 2008a. A barrier nucleosome model for statistical positioning of nucleosomes throughout the yeast genome. Genome Res 18: 1073-1083. doi:10.1101/gr.078261.108

Mavrich TN, Jiang C, Ioshikhes IP, Li X, Venters BJ, Zanton SJ, Tomsho LP, Qi J, Glaser RL, Schuster SC, et al. 2008b. Nucleosome organization in the Drosophila genome. Nature 453: 358-362. doi:10.1038/nature06929

McGhee JD, Sleumer MC, Bilenky M, Wong K, McKay SJ, Goszczynski B, Tian H, Krich ND, Khattra J, Holt RA, et al. 2007. The ELT-2 GATA-factor and the global regulation of transcription in the C. elegans intestine. Dev Biol 302: 627-645. doi:10.1016/j.ydbio.2006.10.024
Ong C-T, Corces VG. 2011. Enhancer function: new insights into the regulation of tissue-specific gene expression. Nat Rev Genet 12: 283-293. doi:10.1038/nrg2957

Osterwalder M, Barozzi I, Tissières V, Fukuda-Yuzawa Y, Mannion BJ, Afzal SY, Lee EA, Zhu Y, Plajzer-Frick I, Pickle CS, et al. 2018. Enhancer redundancy provides phenotypic robustness in mammalian development. Nature 554: 239-243. doi:10.1038/nature25461

Peckham HE, Thurman RE, Fu Y, Stamatoyannopoulos JA, Noble WS, Struhl K, Weng Z. 2007. Nucleosome positioning signals in genomic DNA. Genome Res 17: 1170-1177. doi:10.1101/gr.6101007

Pervouchine DD, Djebali S, Breschi A, Davis CA, Barja PP, Dobin A, Tanzer A, Lagarde J, Zaleski C, See L-H, et al. 2015. Enhanced transcriptome maps from multiple mouse tissues reveal evolutionary constraint in gene expression. Nat Commun 6: 5903. doi:10.1038/ncomms6903

Petrella LN, Wang W, Spike CA, Rechtsteiner A, Reinke V, Strome S. 2011. Synmuv B proteins antagonize germline fate in the intestine and ensure C. elegans survival. Development 138: 1069-1079. doi:10.1242/dev .059501

Pich O, Muiños F, Sabarinathan R, Reyes-Salazar I, Gonzalez-Perez A, LopezBigas N. 2018. Somatic and germline mutation periodicity follow the orientation of the DNA minor groove around nucleosomes. Cell 175: 1074-1087.e18. doi:10.1016/j.cell.2018.10.004

Quillien A, Abdalla M, Yu J, Ou J, Zhu LJ, Lawson ND. 2017. Robust identification of developmentally active endothelial enhancers in zebrafish using FANS-assisted ATAC-Seq. Cell Rep 20: 709-720. doi:10.1016/j .celrep.2017.06.070

Rajendra Sonawane A, Platig J, Fagny M, Chen C-Y, Paulson JN, LopesRamos CM, DeMeo DL, Quackenbush J, Glass K, Kuijjer ML. 2017. Understanding tissue-specific gene regulation. Cell Rep 21: 10771088. doi:10.1016/j.celrep.2017.10.001

$\mathrm{R}$ Core Team. 2019. R: a language and environment for statistical computing. $\mathrm{R}$ Foundation for Statistical Computing, Vienna. https://www.R-project .org/.

Reimand J, Kull M, Peterson H, Hansen J, Vilo J. 2007. g:Profiler-a web-based toolset for functional profiling of gene lists from large-scale experiments. Nucleic Acids Res 35: W193-W200. doi:10.1093/nar/ gkm226

Reinke V, Cutter AD. 2009. Germline expression influences operon organization in the Caenorhabditis elegans genome. Genetics 181: 1219-1228. doi:10.1534/genetics.108.099283

Robinson PJ, Trnka MJ, Bushnell DA, Davis RE, Mattei P-J, Burlingame AL, Kornberg RD. 2016. Structure of a complete mediator-RNA polymerase II pre-initiation complex. Cell 166: 1411-1422.e16. doi:10.1016/j.cel .2016 .08 .050

Saito TL, Hashimoto S-I, Gu SG, Morton JJ, Stadler M, Blumenthal T, Fire A, Morishita S. 2013. The transcription start site landscape of C. elegans. Genome Res 23: 1348-1361. doi:10.1101/gr.151571.112

Satchwell SC, Drew HR, Travers AA. 1986. Sequence periodicities in chicken nucleosome core DNA. J Mol Biol 191: 659-675. doi:10.1016/0022-2836 (86) $90452-3$

Schep AN, Buenrostro JD, Denny SK, Schwartz K, Sherlock G, Greenleaf WJ. 2015. Structured nucleosome fingerprints enable high-resolution mapping of chromatin architecture within regulatory regions. Genome Res 25: $1757-1770$. doi:10.1101/gr.192294.115

Schilbach S, Hantsche M, Tegunov D, Dienemann C, Wigge C, Urlaub H, Cramer P. 2017. Structures of transcription pre-initiation complex with TFIIH and mediator. Nature 551: 204-209. doi:10.1038/ nature 24282

Segal E, Fondufe-Mittendorf $\mathrm{Y}$, Chen L, Thåström A, Field Y, Moore IK, Wang J-PZ, Widom J. 2006. A genomic code for nucleosome positioning. Nature 442: 772-778. doi:10.1038/nature04979

Smith AD, Sumazin P, Zhang MQ. 2007. Tissue-specific regulatory elements in mammalian promoters. Mol Syst Biol 3: 73. doi:10.1038/msb4100114

Spitz F, Furlong EEM. 2012. Transcription factors: from enhancer binding to developmental control. Nat Rev Genet 13: 613-626. doi:10.1038/ nrg3207

Struhl K, Segal E. 2013. Determinants of nucleosome positioning. Nat Struct Mol Biol 20: 2279-2289. doi:10.1038/nsmb.2506

Supek F, Bošnjak M, Škunca N, Šmuc T. 2011. REVIGO summarizes and visualizes long lists of Gene Ontology terms. PLoS One 6: e21800. doi:10 .1371 /journal.pone.0021800

Tolstorukov MY, Kharchenko PV, Goldman JA, Kingston RE, Park PJ. 2009. Comparative analysis of H2A.Z nucleosome organization in the human and yeast genomes. Genome Res 19: 967-977. doi:10.1101/gr.084830 108

Trifonov EN. 1980. Sequence-dependent deformational anisotropy of chromatin DNA. Nucleic Acids Res 8: 4041-4054. doi:10.1093/nar/8.17.4041

Wang J-PZ, Widom J. 2005. Improved alignment of nucleosome DNA sequences using a mixture model. Nucleic Acids Res 33: 6743-6755 doi:10.1093/nar/gki977 


\section{C. elegans regulatory architectures}

White RJ, Collins JE, Sealy IM, Wali N, Dooley CM, Digby Z, Stemple DL, Murphy DN, Billis K, Hourlier T, et al. 2017. A high-resolution mRNA expression time course of embryonic development in zebrafish. eLife 6: e30860. doi:10.7554/eLife.30860

Whyte WA, Orlando DA, Hnisz D, Abraham BJ, Lin CY, Kagey MH, Rahl PB, Lee TI, Young RA. 2013. Master transcription factors and mediator establish super-enhancers at key cell identity genes. Cell 153: 307-319. doi:10.1016/j.cell.2013.03.035

Widom J. 2001. Role of DNA sequence in nucleosome stability and dynamics. Q Rev Biophys 34: 269-324. doi:10.1017/S0033583501003699

Wright GM, Cui F. 2019. The nucleosome position-encoding WW/SS sequence pattern is depleted in mammalian genes relative to other eukaryotes. Nucleic Acids Res 47: 7942-7954. doi:10.1093/nar/gkz544
Wu X, Shi Z, Cui M, Han M, Ruvkun G. 2012. Repression of germline RNAi pathways in somatic cells by retinoblastoma pathway chromatin complexes. PLoS Genet 8: e1002542. doi:10.1371/journal.pgen .1002542

Yu G, Wang L-G, Han Y, He Q-Y. 2012. clusterProfiler: an R package for comparing biological themes among gene clusters. OMICS 16: 284-287. doi:10.1089/omi.2011.0118

Zhurkin VB, Lysov YP, Ivanov VI. 1979. Anisotropic flexibility of DNA and the nucleosomal structure. Nucleic Acids Res 6: 1081-1096. doi:10.1093/ nar/6.3.1081

Received May 12, 2020; accepted in revised form October 8, 2020. 


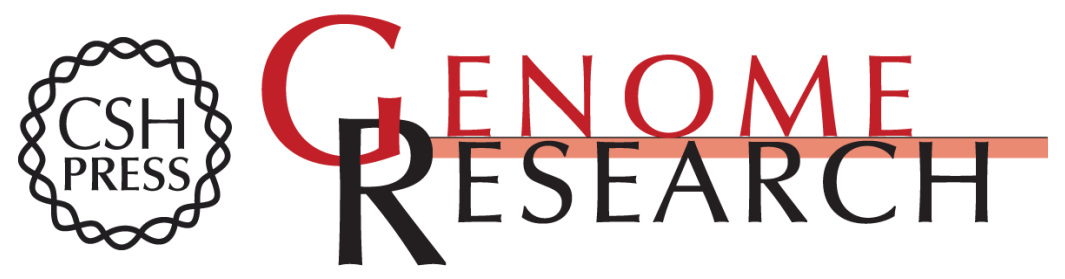

\section{Distinctive regulatory architectures of germline-active and somatic genes in C. elegans}

Jacques Serizay, Yan Dong, Jürgen Jänes, et al.

Genome Res. 2020 30: 1752-1765 originally published online October 22, 2020

Access the most recent version at doi:10.1101/gr.265934.120

Supplemental Material

References

Open Access

Creative Commons

License

Email Alerting Service
http://genome.cshlp.org/content/suppl/2020/11/16/gr.265934.120.DC1

This article cites 82 articles, 19 of which can be accessed free at: http://genome.cshlp.org/content/30/12/1752.full.html\#ref-list-1

Freely available online through the Genome Research Open Access option.

This article, published in Genome Research, is available under a Creative Commons License (Attribution 4.0 International), as described at http://creativecommons.org/licenses/by/4.0/.

Receive free email alerts when new articles cite this article - sign up in the box at the top right corner of the article or click here.

\section{Affordable, Accurate Sequencing.}

To subscribe to Genome Research go to: https://genome.cshlp.org/subscriptions 